\title{
Time Reversal Based Active Array Source Localization
}

\author{
Foroohar Foroozan, Student Member, IEEE, and Amir Asif, Senior Member, IEEE
}

\begin{abstract}
Source localization especially direction-of-arrival (DOA) estimation using sensor arrays is of considerable interest in both classical array signal processing and radar applications. Most radar systems are designed under the line-of-sight (LOS) assumption with multipath echos treated as undesired clutter noise. Strong multipath, therefore, has a negative impact on the resolution of the radar systems and their ability in accurately localizing the target. Rather than treating multipath as a detrimental effect, the paper introduces time reversal (TR) to exploit spatial/multipath diversity in improving the capability of the existing localization algorithms. In particular, we design TR based range and DOA estimators that adaptively adjust the probing radar waveforms to the multipath characteristics of the environment. The benefits of the spatial/multipath diversity in the proposed DOA and range estimators are quantified by deriving the respective Cramér-Rao bounds (CRB) and comparing them with the analytical expressions for their conventional counterparts. Numerical simulations also confirm the benefit of applying TR to source localization algorithms especially at low signal-to-noise ratios below $-5 \mathrm{~dB}$.
\end{abstract}

Index Terms-Array signal processing, Cramér-Rao bounds, direction-of-arrival (DOA) estimation, multipath, range estimation, source localization, time reversal.

\section{INTRODUCTION}

A $\mathrm{N}$ important problem in array signal processing is the estimation of the location (namely, the range $(R)$, azimuth $(\psi)$, and elevation $(\theta)$ in 3-D polar coordinates) of a point target illuminated externally. Such localization techniques in which the medium (that is, the channel encompassing the transmitting/receiving antenna array ${ }^{1}$ and the passive target) is probed by an external source to produce an echo (backscatter), which is then analyzed to determine the location of the target, are referred to as active localization algorithms. The target's range $R$ is typically estimated from the return time of travel of the backscatters of the probing signal observed at the antenna

Manuscript received January 07, 2011; revised March 05, 2011; accepted March 05, 2011. Date of publication March 14, 2011; date of current version May 18, 2011. The associate editor coordinating the review of this manuscript and approving it for publication was Prof. Dominic K. C. Ho.

F. Foroozan is with the Faculty of Engineering and Applied Science, University of Ontario Institute of Technology (UOIT), Oshawa, Canada (e-mail: foroozan@cse.yorku.ca).

A. Asif is with the Department of Computer Science and Engineering, York University, Toronto, M3J 1P3, Canada (e-mail: asif@cse.yorku.ca).

Color versions of one or more of the figures in this paper are available online at http://ieeexplore.ieee.org.

Digital Object Identifier 10.1109/TSP.2011.2128317

${ }^{1}$ In most radar applications, a single antenna array is used to probe the channel as well as to record its backscatters though the theory is readily generalizable to a pair of transmitting and receiving antenna arrays. array, while the azimuth and elevation angles $\{\psi, \theta\}$ are derived from the direction-of-arrival (DOA) of the backscatters [1], [2]. Without a direct line of sight between the antenna array and the target, the backscatter observations have a significant time spread with their constituent signal components traveling along multiple paths differing in arrival times, amplitudes, and DOAs. Multipath, therefore, impacts the performance of the conventional localization algorithms [3]-[5] negatively and the radar performance deteriorates especially at low signal-to-noise ratios (SNR). Consequently, most localization techniques attempt to eliminate the effect of multipath using channel equalization or another form of deconvolution with the approximated channel impulse response.

In this paper, we take a different approach and address the question of what can be gained if a localization estimator exploits the additional information existing in the temporal structure of the observed fields (i.e., in the set of multipath delays) to its advantage. Only a handful of array processing approaches [6]-[8] treat multipath positively. While [6] focuses on the theoretical Cramér-Rao bounds (CRB) defining the types of gains possible with positive treatment of multipath, publication [7] develops a range estimator for a rich scattering environment with unknown multipath parameters based only on the forward propagation step without introducing any TR, and [8] extends TR to imaging applications in an acoustic environment. This paper applies TR array processing to the direct estimation of the DOA for radar-a problem not previously addressed in the context of TR in the aforementioned references or elsewhere. Our approach is based on the TR [9]-[13] setup shown in Fig. 1 and does not assume any particular multipath model. An active antenna array receives the superposition of several attenuated and delayed replicas of the backscattered field from a passive target. We assume that the target and sensor arrays are far apart such that the far-field approximation is applicable, have no relative motion, and observations are recorded by a single, linear multielement sensor array with known geometry and spatial locations. The conventional range and/or DOA estimation algorithms use this set of observations to localize the target. In the proposed TR setup, the backscatter observations made by the antenna array are digitized, energy normalized, time-reversed, and retransmitted to probe the channel a second time. The backscatters of the time-reversed signals are used by the TR localization algorithms. Our intuition is that TR is equivalent to waveform adaptation, tailoring the retransmitted signals to the channel characteristics and the target's scattering, thus, providing improved performance.

To quantify the performance of the TR localization algorithms, we derive analytical expressions for the CRBs of the TR range and DOA estimators. The TR CRBs are compared 


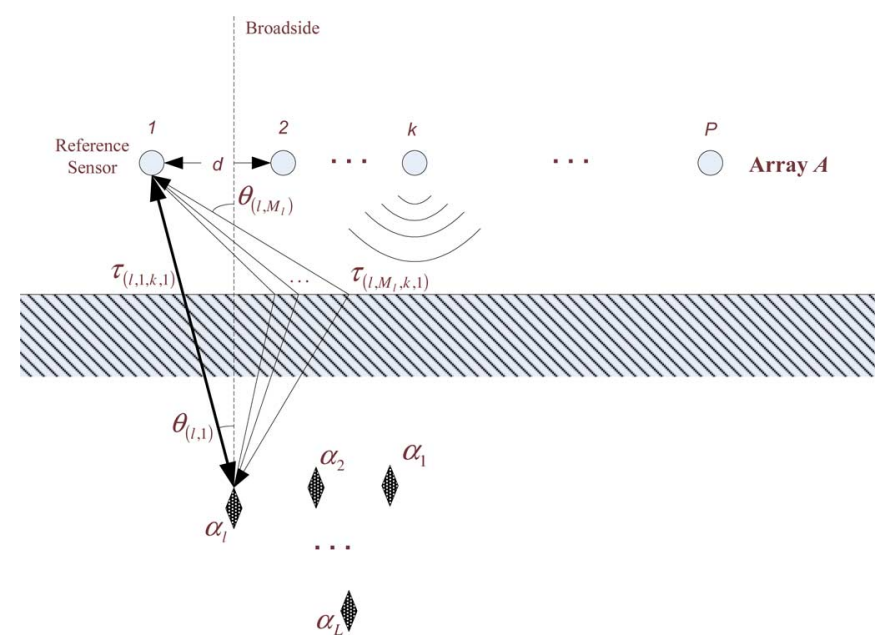

Fig. 1. Experimental setup for the TR localization estimator. Element $k$ of Array $A$ probes the channel. Backscatters from targets (denoted by " $\diamond$ ") travel via multiple paths before reaching Array $A$. Notation $\tau_{(l, i, k, j)}$ is the delay associated with the backscatter component reflected from target $l$ traveling via path $i$ between the probing element $k$ and recording element $j$. The DOA of the backscatter from target $l$ via path $i$ is denoted by $\theta_{(l, i)}$.

with the CRBs for the conventional localization algorithms that use only the observations made during the forward propagation step. In addition, actual performance characteristics of the TR and conventional estimators are plotted for different SNRs to illustrate the superiority of the TR localization. In both cases, the TR algorithms outperform the conventional estimators by a gain of up to $6 \mathrm{~dB}$ at SNRs below $-5 \mathrm{~dB}$. A number of practical applications including ultra-wideband radar, deep space communications, seismology, deep water acoustics, and medical imaging operate at such low SNRs. The major contributions of the paper are as follows.

1) Use TR to exploit the spatial diversity arising from multipath propagation: We formulate the TR observations in terms of range (or, alternatively, as the direct path time delay) and DOA (interelements delays) of $L$ passive targets when a wideband signal is used to probe a rich multipath environment in the forward probing step. TR probes the environment a second time using the time reversed versions of the backscatters observed during the forward probing step as probing signals.

2) Derive TR range and DOA estimation algorithms: The framework derived in item 1) is used to develop TR based localization algorithms designed for rich multipath environments. The TR range estimator (presented previously for a single pair of antenna elements in [13] and extended here to multielement antenna arrays) is based on the maximum likelihood approach that uses the TR set of observations, while the TR/DOA estimator is based on the wideband Capon [14]-[16]. In other words, the wideband observations are decomposed into many narrowband bins using filter banks or the discrete Fourier transform before applying the narrowband Capon to each bin. The objective of the paper is not so much to devise a DOA estimation procedure based on Capon but to quantify the improvement, if any, with the application of TR to DOA estimators. Since the focus of this paper is on the
TR/DOA estimator, the TR range estimator is only briefly introduced for the purpose of completeness.

3) Compare TR range/DOA estimation algorithms with their conventional counterparts: We derive analytical expressions for the CRBs of TR range and DOA estimation algorithms for localizing $L$ passive targets buried in a rich multipath environment. The TR/DOA CRBs are compared with those of the conventional DOA estimation approaches. Further, the actual performances of the TR and conventional localization algorithms are compared using numerical simulations. Unlike [13], where the CRB for the TR range estimator was derived for a 2-multipath model with a single target, we generalize the result for $M$-multipath and $L$ targets in this paper. The CRB result for TR DOA estimator is a new result.

Our conclusive results are as follows:

1) The CRBs providing the lower bounds for the error covariances of the TR range and DOA estimators are substantially less than those of the conventional localization algorithms illustrating the potential of better performance with the TR setup. TR introduces an additional step where the time reversed waveforms probe the channel a second time. Intuitively, this leads to the inherent adaptation of the probing waveform to the channel characteristics, thereby, resulting in a better performance. By exploiting multipath diversity, the TR/DOA estimator introduces beamspace beamforming (a built-in feature attributed to TR) to focus the probing signal towards the target.

2) Monte Carlo simulations verify that the TR localization algorithms estimate the range and DOA of a target in a rich clutter environment with higher accuracy than the conventional approaches.

3) The pseudospectrum of the TR localization algorithms have a narrower mainlobe and smaller sidelobes, thus, providing a higher resolution capability.

In terms of organization, Section II provides the background material needed to follow the rest of the article. Section III develops the framework for both the conventional and TR based DOA estimators, while Section IV presents the TR range and DOA estimators. Section V derives the CRBs in terms of error variances of the estimated target's location and DOA obtained from the conventional and TR localization estimators. Section VI compares the performances of the two DOA estimators in terms of the CRBs as well as the actual performance characteristics obtained from Monte Carlo simulations. Finally, Section VII concludes the paper.

\section{BACKGROUND}

\section{A. List of Symbols}

The list of commonly used symbols is as follows. Additional notation, where needed, is introduced in the relevant sections.

\section{Symbol Notation}

CRB Cramér-Rao Bound.

DOA Direction-of-arrival.

$\operatorname{tr}\{\cdot\} \quad$ Trace of the matrix enclosed in parenthesis.

$\boldsymbol{a}(\boldsymbol{\theta}) \quad$ Array response vector for DOA $\boldsymbol{\theta}$. 


$\begin{array}{ll}\boldsymbol{\alpha} & \text { Unknown vector parameter to be estimated. } \\ \mathbf{C}_{r}(\omega) & \text { PSD matrix of the observations } \mathbf{r}\left(t_{n}\right) . \\ D & \text { Length of unknown vector parameter } \boldsymbol{\alpha} . \\ H & \text { Conjugate transpose if appearing as a superscript. } \\ \mathcal{I}(\boldsymbol{\alpha}) & \text { Fisher information matrix (FIM) for } \boldsymbol{\alpha} . \\ \mathbf{K}_{n}(\boldsymbol{\alpha}) & \begin{array}{l}\text { Covariance matrix of order } P \text { for observations } \\ \mathbf{r}\left(t_{n}\right) .\end{array} \\ L & \text { Total number of targets. } \\ M_{l} & \text { Number of paths for target } l . \\ \boldsymbol{\mu}_{n}(\boldsymbol{\alpha}) & \text { Mean vector of length } P \text { for observations } \mathbf{r}\left(t_{n}\right) . \\ N & \text { Total number of snapshots. } \\ P & \text { Total number of elements in the antenna array. } \\ \Re\{.\} & \text { Real part of the parameter enclosed in parenthesis. } \\ \mathbf{r}\left(t_{n}\right) & \text { Observation vector of length } P \text { from the forward } \\ & \text { probing stage. } \\ * & \text { Complex conjugation if appearing as a superscript. }\end{array}$

\section{B. On Cramér-Rao Bound}

Assume that the vector parameter $\boldsymbol{\alpha}=\left\{\alpha_{i}\right\}$ of length $D$ is to be estimated from a $P$-dimensional observation vector $\mathbf{r}_{n} \triangleq$ $\mathbf{r}\left(t_{n}\right),(1 \leq n \leq N, N$ being the total number of snapshots) having the complex Gaussian distribution

$$
\begin{aligned}
p_{\mathbf{r}_{n} \mid \boldsymbol{\alpha}}\left(\mathbf{r}_{n}\right) & =\frac{1}{\operatorname{det}\left[\pi \mathbf{K}_{n}(\boldsymbol{\alpha})\right]} \\
\times & \times \exp \left\{-\left(\mathbf{r}_{n}^{H}-\boldsymbol{\mu}_{n}^{H}(\boldsymbol{\alpha})\right) \mathbf{K}_{n}^{-1}(\boldsymbol{\alpha})\left(\mathbf{r}_{n}-\boldsymbol{\mu}_{n}(\boldsymbol{\alpha})\right)\right\}
\end{aligned}
$$

where $\boldsymbol{\mu}_{n}$ denotes the mean and $\mathbf{K}_{n}$ is the covariance matrix. Note that both mean $\boldsymbol{\mu}_{n}$ and covariance $\mathbf{K}_{n}$ in (1) are functions of the unknown vector parameter $\boldsymbol{\alpha}$. It is well known [2], [17] that the CRB provides a lower bound on the covariance of any unbiased estimator of $\boldsymbol{\alpha}$ and is given by the inverse of the FIM, i.e.,

$$
\operatorname{var}\left[\hat{\alpha}_{i}-\alpha_{i}\right] \geq[\mathrm{CRB}(\boldsymbol{\alpha})]_{i i}=\left[\mathcal{I}(\boldsymbol{\alpha})^{-1}\right]_{i i}
$$

for $(1 \leq i \leq D)$. In (2), $\mathcal{I}(\boldsymbol{\alpha})$ is the square FIM for vector $\boldsymbol{\alpha}$ with dimension $D$. Based on a complex Gaussian observation model (1), the asymptotic values [18] for the entries of the FIM are given by the following expression

$$
\begin{aligned}
& {[\mathcal{I}(\boldsymbol{\alpha})]_{i j} } \approx \underbrace{\frac{N}{\pi} \Re\left\{\int \mathbf{d}_{i}^{H}\left(e^{\hat{\jmath} \omega}, \boldsymbol{\alpha}\right) \mathbf{C}_{r}^{-1}\left(e^{\hat{\jmath} \omega}\right) \mathbf{d}_{j}\left(e^{\hat{\jmath} \omega}, \boldsymbol{\alpha}\right) d \omega\right\}}_{\text {Term } 3.1} \\
&+\underbrace{\frac{N}{2 \pi} \int \operatorname{tr}\left\{\frac{\partial \mathbf{C}_{r}\left(e^{\hat{\jmath} \omega}\right)}{\partial \alpha_{i}} \mathbf{C}_{r}^{-1}\left(e^{\hat{\jmath} \omega}\right) \frac{\partial \mathbf{C}_{r}\left(e^{\hat{\jmath} \omega}\right)}{\partial \alpha_{j}} \mathbf{C}_{r}^{-1}\left(e^{\hat{\jmath} \omega}\right)\right\} d \omega}_{\text {Term } 3.2}
\end{aligned}
$$

where $N$ is the total number of snapshots, assumed large for the approximation to hold true and $\hat{\jmath}=\sqrt{-1}$. Notation $\mathbf{C}_{r}\left(e^{\hat{\jmath} \omega}\right)$ is the power spectral density (psd) matrix of the observations $\mathbf{r}\left(t_{n}\right)$. The entries of the vector $\mathbf{d}_{i}\left(e^{\hat{\jmath} \omega}, \boldsymbol{\alpha}\right)$ are the discrete time Fourier transform (DTFT) of the partial derivative of the mean $\boldsymbol{\mu}(\boldsymbol{\alpha})$ with respect to the unknown parameters $\alpha_{i}$, for $(1 \leq i \leq$ $D)$ and are defined as follows:

$$
\mathbf{d}_{i}\left(e^{\hat{\jmath} \omega}, \boldsymbol{\alpha}\right)=\operatorname{DTFT}\left\{\frac{\partial \boldsymbol{\mu}_{n}(\boldsymbol{\alpha})}{\partial \alpha_{i}}\right\}
$$

In this paper, our focus is on active localization with passive, deterministic targets buried in a multipath environment. In such cases, the psd $\mathbf{C}_{r}\left(e^{\hat{\jmath} \omega}\right)$ is independent of the unknown parameter $\boldsymbol{\alpha}$. Term 3.2 (referred to as the Whittle's formula [19]), therefore, reduces to 0 . As such, Term 3.1 is of importance to us and is used in deriving the CRB.

\section{On Time Reversal}

Time reversal (TR) approaches have been applied to a wide range of signal processing applications in acoustics, radiotherapy medicine, geophysics and geoscience devices, radar systems, and wireless networks. With an increased availability of radio frequency $(\mathrm{RF})$ instruments, there is a growing research effort to apply TR to the electromagnetic (EM) domain. The TR EM applications include wireless communications as well as target detection and localization in radar surveillance. For a complete list of applications and history of TR, the readers are referred to the TR array imaging survey paper [20]. In a typical TR experiment, a short pulse transmitted by a source through a high scattering medium is received by an array, then time reversed, energy normalized, and retransmitted through the same medium. If the scattering channel is reciprocal and rich in multipath, the time reversed signals travel backwards through the background medium and undergo similar changes (such as multiple scattering, reflections, and refraction) that they underwent in the forward direction step and finally refocuses on the original source. In the array processing terminology, the TR array is analogous to a convex lens with an effective aperture that is larger than that of the aperture of the physical array, thereby, focusing all available multipaths at the location of the target. In a homogeneous medium, the focusing resolution of TR is bounded by the classical diffraction limit (Rayleigh resolution) [21]. But in high scattering environment, the focusing spot size introduced by TR is much narrower which beats the Rayleigh resolution limit and is referred to as super-resolution focusing capability of TR [22], [23]. This phenomena is attractive for many applications such as target localization in radar systems, which is the topic of the discussion in this paper. In the following section, we formulate the TR framework used later for developing the TR localization algorithms.

As a side note to our discussion on TR, we observe that though the TR step can intuitively be carried out either physically or computationally, its physical implementation is more suitable for low SNR applications as is the case for the localization algorithms considered in this paper.

\section{SYSTEM MODEL}

In this section, we introduce the observation model (as well as the underlying assumptions) used in the article during the forward and TR probing steps of the estimation algorithms. A 
known complex bandpass signal $f(t) \exp \left(\hat{\jmath} \omega_{c} t\right)\left(\omega_{c}\right.$ being the angular carrier frequency), transmitted by element $k$ of Array $A$, is backscattered by the targets (shown in Fig. 1 as ' $\diamond$ ' with spatial location $\boldsymbol{\alpha}_{l}$, for $\left.(1 \leq l \leq L)\right)$. The $P$-element antenna array (Array $A$ ) receives a sum of scaled, time delayed echos of the probing signal through multiple paths resulting from among other factors the clutter and/or the medium boundaries. Following signal processing theory (rather than electromagnetic scattering theory), the backscatters of the probing signal received at element $j$ of array $A$ after down conversion to baseband and sampling are given as

$r_{(k, j)}(t)=\sum_{l=1}^{L} \sum_{i=1}^{M_{(l)}} X_{(l, i, k, j)} f\left(t-\tau_{(l, i, k, 1)}-\Delta \tau_{(l, i, j)}\right)+v_{(k, j)}(t)$

for $\left(t \in\left\{t_{1}, \ldots, t_{N}\right\}\right)$. Subscript $(l, i, k, j)$ in (5) represents the backscatter component associated with target $l$ traveling via path $i$ when element $k$ of Array $A$ (denoted by $A_{k}$ ) probes the channel and the observation is recorded by element $j$ (denoted by $A_{j}$ ). Explicitly, the symbols used in (5) and a few others introduced later are defined below.

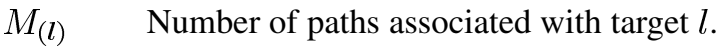

$X_{(l, i, k, j)}$ Attenuation associated with backscatter component reflected from target $l$ traveling via path $i$ with element $k$ probing and element $j$ receiving.

$\boldsymbol{\theta}_{(l, i)} \quad$ DOA of the backscatter from target $l$ propagating via path $i$.

$\boldsymbol{a}\left(\boldsymbol{\theta}_{(l, i)}\right) \quad(P \times 1)$ array response to a plane wave reflected from the $l$ th target propagating via path $i$.

$\tau_{(l, i, k, 1)} \quad$ Reference delay associated with backscatter from target $l$ traveling via path $i$ with element $k$ probing element 1 receiving.

$\Delta \tau_{(l, i, j)}$ Interelement delay associated with path $i$ originating from target $l$ in excess of $\tau_{(l, i, k, 1)}$ with respect to the receiving element $j$.

$\boldsymbol{v}_{k}(t) \quad$ Observation noise vector with element $k$ transmitting.
Since the focus of the paper is on colocated antennas, therefore, we assume that $X_{(l, i, k, j)}=X_{(l, i)}$, i.e., the attenuation factor from target $l$ via path $i$ is the same for accompanying backscatters recorded at different elements in the antenna array provided they are reflected from the same target $l$ and propagate via the same path $i$. The order $M_{(l)}$ of the multipath $l$ used in the system formulation is not used in our localization algorithm nor is required for computing the CRB. However, the number $L$ of targets is estimated prior to the proposed localization algorithm and is assumed known. Estimating the number of targets is a traditional problem in array signal processing and is based on decomposing the channel response matrix (defined later) into signal-plus-noise and noise-only subspaces using singular value decomposition (SVD). The number of targets is then equal to the dimension of the signal-plus-noise subspace, which can be estimated using either decision theoretic approaches such as the sphericity test [24], or information theoretic approaches such as the Akaike information criterion (AIC) [25] and the minimum description length (MDL) [26], [27]. A number of localization algorithms involve a similar step where the number of targets is determined before the range and DOA estimation.

In the frequency domain, (5) can be expressed as

$$
\begin{gathered}
R_{(k, j)}\left(e^{\hat{\jmath} \omega}\right)=\sum_{l=1}^{L} \sum_{i=1}^{M_{(l)}} e^{-\hat{\jmath} \omega \tau_{(l, i, k, 1)}} X_{(l, i)} e^{-\hat{\jmath} \omega \Delta \tau_{(l, i, j)}} F\left(e^{\hat{\jmath} \omega}\right) \\
+V_{(k, j)}\left(e^{\hat{\jmath} \omega}\right) .
\end{gathered}
$$

Using the vector-matrix notation, (6) can be represented as (7), shown at the bottom of the page, which is further expressed in the compact form as

$$
\mathbf{r}_{k}\left(e^{\hat{\jmath} \omega}\right)=A(\boldsymbol{\Theta}) \boldsymbol{X} \boldsymbol{\Gamma}_{k}\left(e^{\hat{\jmath} \omega}\right) F\left(e^{\hat{\jmath} \omega}\right)+\mathbf{v}_{k}\left(e^{\hat{\jmath} \omega}\right)
$$

For a uniform linear array (ULA), notation $\boldsymbol{A}(\boldsymbol{\Theta})$ is defined as a $(P \times M)$ matrix containing all possible $M$-steering vectors (with $M$ being the total number of multipaths for all $L$ targets, i.e., $\left.M=\sum_{l=1}^{L} M_{(l)}\right)$ and is given by (9), shown at the bottom of the next page; $\boldsymbol{X}$ as a $(M \times M)$ diagonal matrix with attenuation factors ordered in terms of the target number and number of multipath as its diagonal entries, and $\Gamma_{k}\left(e^{\hat{\jmath} \omega}\right)$ as a $(M \times 1)$ vector of the delays with respect to the reference element 1 , for $(1 \leq l \leq L)$, again ordered in terms of the target number and

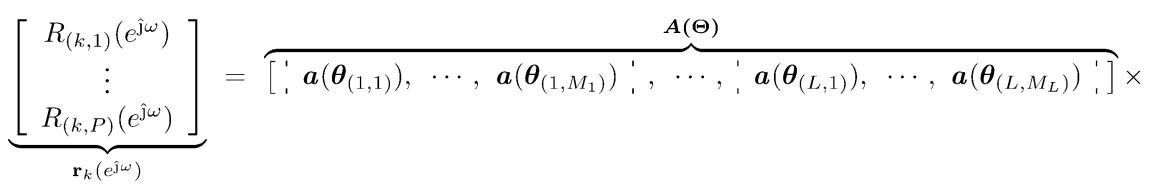

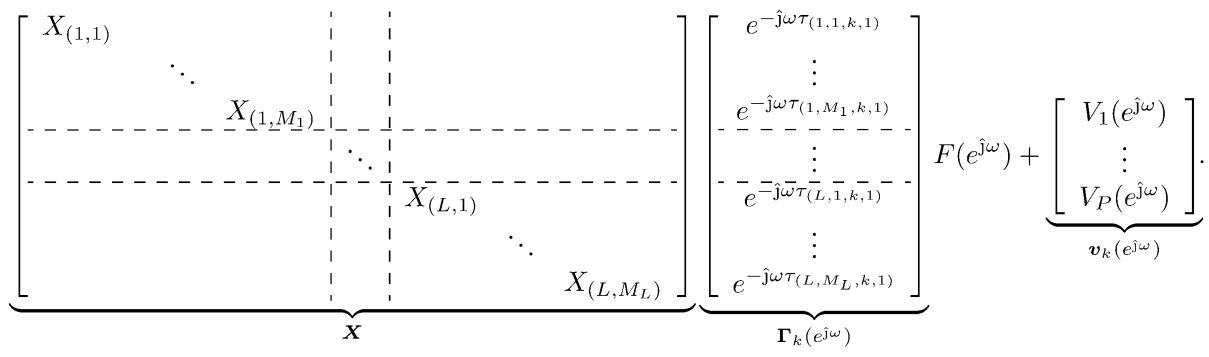


number of multipath. Next, we define the channel response matrix for the channel being probed as

$$
\forall k, j=1, \ldots, P, \mathbf{H}\left(e^{\hat{\jmath} \omega}\right) \triangleq\left\{H\left(e^{\hat{\jmath} \omega} ; A_{j} \leftarrow A_{k}\right)\right\} .
$$

Note that $\mathbf{H}\left(e^{\hat{\jmath} \omega}\right)$ is the $(P \times P)$ channel response matrix with constituent elements

$$
H_{j k}\left(e^{\hat{\jmath} \omega} ; A_{j} \leftarrow A_{k}\right)=\sum_{l=1}^{L} \sum_{i=1}^{M_{(l)}} X_{(l, i)} \boldsymbol{a}_{j}\left(\boldsymbol{\theta}_{(l, i)}\right) e^{-\hat{\jmath} \omega \tau_{(l, i, k, 1)}}
$$

where $\boldsymbol{a}_{j}\left(\boldsymbol{\theta}_{(l, i)}\right)$ refers to the $j$ th element of the $(P \times 1)$ array response vector $\boldsymbol{a}\left(\boldsymbol{\theta}_{(l, i)}\right)$. The matrix $\mathbf{H}\left(e^{\hat{\jmath} \omega}\right)$ consists of $P$ column vectors defined as follows:

$$
\mathbf{H}\left(e^{\hat{\jmath} \omega}\right)=\left[\mathbf{h}_{1}\left(e^{\hat{\jmath} \omega}\right), \mathbf{h}_{2}\left(e^{\hat{\jmath} \omega}\right), \ldots, \mathbf{h}_{P}\left(e^{\hat{\jmath} \omega}\right)\right]
$$

where each vector $\mathbf{h}_{k}\left(e^{\hat{\jmath} \omega}\right)=\left[H_{1 k}\left(e^{\hat{\jmath} \omega}\right), \ldots, H_{P k}\left(e^{\hat{\jmath} \omega}\right)\right]^{T}$. Based on the above definition of $\mathbf{H}\left(e^{\hat{\jmath} \omega}\right)$, (8) is expressed in the alternate form as

$$
\begin{aligned}
\mathbf{r}_{k}\left(e^{\hat{\jmath} \omega}\right) & =\mathbf{h}_{k}\left(e^{\hat{\jmath} \omega}\right) F\left(e^{\hat{\jmath} \omega}\right)+\mathbf{v}_{k}\left(e^{\hat{\jmath} \omega}\right) \\
& =\mathbf{H}\left(e^{\hat{\jmath} \omega}\right) \mathbf{e}_{k} F\left(e^{\hat{\jmath} \omega}\right)+\mathbf{v}_{k}\left(e^{\hat{\jmath} \omega}\right)
\end{aligned}
$$

where $\mathbf{e}_{k}$ is a $(P \times 1)$ vector of zero entries except for the $k$ th element, which is 1 , and $\mathbf{v}_{k}\left(e^{j \omega}\right) \sim \mathcal{C N}\left(\mathbf{0}, \sigma_{v}^{2} \mathbf{I}_{P}\right)$ is the accumulated noise. Recall that $k$ corresponds to the array element that probed the medium initially. It is worth noting that the channel response vector is expressed in terms of the time delays, attenuation factors, and DOAs of both the direct path as well as multipath as

$$
\mathbf{h}_{k}\left(e^{\hat{\jmath} \omega}\right)=\boldsymbol{A}(\boldsymbol{\Theta}) \boldsymbol{X} \boldsymbol{\Gamma}_{k}\left(e^{\hat{\jmath} \omega}\right) .
$$

Following the principle of TR, the recorded signal $\mathbf{r}_{k}\left(e^{\hat{\jmath} \omega}\right)$ is energy normalized by a constant factor of $g$, time reversed (equivalent to phase conjugation in the frequency domain), and retransmitted back into the medium. Assuming the same multipath propagation environment as in the forward probing step and denoting the TR probing signal vector by $\mathbf{z}\left(e^{\hat{\jmath} \omega}\right)=g \mathbf{r}_{k}^{*}\left(e^{\hat{\jmath} \omega}\right)$, the TR observation vector $\mathbf{p}_{k}\left(e^{\hat{\jmath} \omega}\right)$ is given by

$$
\mathbf{p}_{k}\left(e^{\hat{\jmath} \omega}\right)=\sum_{j=1}^{P} \boldsymbol{A}(\boldsymbol{\Theta}) \boldsymbol{X} \boldsymbol{\Gamma}_{j}\left(e^{\hat{\jmath} \omega}\right)\{\mathbf{z}\}_{(j)}\left(e^{\hat{\jmath} \omega}\right)+\boldsymbol{\zeta}_{k}\left(e^{\hat{\jmath} \omega}\right)
$$

where $\{\mathbf{z}\}_{(j)}\left(e^{\hat{\jmath} \omega}\right)$ refers to the $j$ th entry of the TR probing vector $\mathbf{z}\left(e^{\hat{\jmath} \omega}\right)$ and $\zeta_{k}\left(e^{\hat{\jmath} \omega}\right)$ denotes noise with a covariance of $\sigma_{\zeta}^{2} \boldsymbol{I}_{P}$. The energy normalization factor $\left.g=\sqrt{\left(\left\|F\left(e^{\hat{\jmath} \omega}\right)\right\|^{2}\right) /\left(\left\|\mathbf{r}_{k}\left(e^{\hat{\jmath} \omega}\right)\right\|^{2}\right.}\right)$. In the vector-matrix format, the TR observation is expressed as (16), where the $(M \times P)$ matrix $\mathbf{\Upsilon}\left(e^{\hat{\jmath} \omega}\right)$ represents all the delay components $\left(\boldsymbol{\Gamma}_{j}\left(e^{\hat{\jmath} \omega}\right)\right)$ for $1 \leq j \leq P$.

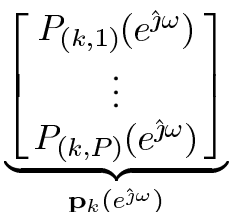

$$
\begin{aligned}
& =\boldsymbol{A}(\boldsymbol{\Theta}) \boldsymbol{X} \underbrace{\left[\begin{array}{llllll}
\boldsymbol{\Gamma}_{1}\left(e^{\hat{\jmath} \omega}\right)\left|\boldsymbol{\Gamma}_{2}\left(e^{\hat{\jmath} \omega}\right)\right| & \cdots & \boldsymbol{\Gamma}_{P}\left(e^{\hat{\jmath} \omega}\right)
\end{array}\right]}_{\boldsymbol{\Upsilon}\left(e^{\hat{\jmath} \omega}\right)}
\end{aligned}
$$

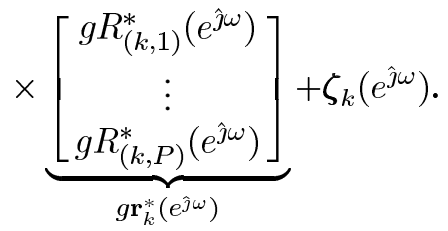

In terms of the channel response matrix $\mathbf{H}\left(e^{\hat{\jmath} \omega}\right)$, the backscattered TR signal at Array $A$ is given by

$$
\begin{aligned}
\mathbf{p}_{k}\left(e^{\hat{\jmath} \omega}\right) & =g \mathbf{H}\left(e^{\hat{\jmath} \omega}\right) \mathbf{r}_{k}^{*}\left(e^{\hat{\jmath} \omega}\right)+\boldsymbol{\zeta}_{k}\left(e^{\hat{\jmath} \omega}\right) \\
& =g \mathbf{H}\left(e^{\hat{\jmath} \omega}\right)\left[\mathbf{H}^{*}\left(e^{\hat{\jmath} \omega}\right) \mathbf{e}_{k} F^{*}\left(e^{\hat{\jmath} \omega}\right)+\mathbf{v}_{k}^{*}\left(e^{\hat{\jmath} \omega}\right)\right]+\boldsymbol{\zeta}_{k}\left(e^{\hat{\jmath} \omega}\right) .
\end{aligned}
$$

Assuming that the effect of the forward step observation noise $\mathbf{v}_{k}\left(e^{\hat{\jmath} \omega}\right)$ and TR observation noise $\zeta_{k}\left(e^{\hat{\jmath} \omega}\right)$ are accumulated as $\mathbf{w}_{k}\left(e^{\hat{\jmath} \omega}\right),(17)$ is expressed as

$$
\begin{aligned}
\mathbf{p}_{k}\left(e^{\hat{\jmath} \omega}\right) & =g \mathbf{T}\left(e^{\hat{\jmath} \omega}\right) \mathbf{e}_{k} F^{*}\left(e^{\hat{\jmath} \omega}\right)+\mathbf{w}_{k}\left(e^{\hat{\jmath} \omega}\right) \\
& =g \mathbf{t}_{k}\left(e^{\hat{\jmath} \omega}\right) F^{*}\left(e^{\hat{\jmath} \omega}\right)+\mathbf{w}_{k}\left(e^{\hat{\jmath} \omega}\right)
\end{aligned}
$$

where $\mathbf{T}\left(e^{\hat{\jmath} \omega}\right)=\mathbf{H}\left(e^{\hat{\jmath} \omega}\right) \mathbf{H}^{*}\left(e^{\hat{\jmath} \omega}\right)$ is the so called TR matrix, and the accumulated noise $\mathbf{w}_{k}\left(e^{\hat{\jmath} \omega}\right) \sim \mathcal{C N}\left(\mathbf{0}, \sigma_{w}^{2} \mathbf{I}_{P}\right)$. The structure of $\mathbf{T}\left(e^{\hat{\jmath} \omega}\right)$ can be expressed in terms of the forward channel responses $H_{i k}$ as

$$
\mathbf{T}\left(e^{\hat{\jmath} \omega}\right)=\left[\mathbf{t}_{1}\left(e^{\hat{\jmath} \omega}\right), \mathbf{t}_{2}\left(e^{\hat{\jmath} \omega}\right), \ldots, \mathbf{t}_{P}\left(e^{\hat{\jmath} \omega}\right)\right]
$$

where each vector $\mathbf{t}_{k}\left(e^{\hat{\jmath} \omega}\right)=\left[T_{1 k}\left(e^{\hat{\jmath} \omega}\right), \ldots, T_{P k}\left(e^{\hat{\jmath} \omega}\right)\right]^{T}$, and the component $T_{n k}\left(e^{\hat{\jmath} \omega}\right)=\sum_{i=1}^{P} H_{n i}\left(e^{\hat{\jmath} \omega}\right) H_{i k}^{*}\left(e^{\hat{\jmath} \omega}\right)$.

Using the notation derived in (5)-(19), the following section explains both the conventional and TR range and DOA estimators for localizing $L$ passive targets buried in a high multipath clutter environment.

$$
\boldsymbol{A}(\boldsymbol{\Theta})=\left[\begin{array}{ccccc}
1 & \cdots & 1 & \cdots & 1 \\
\vdots & \ddots & \vdots & \vdots & \\
\exp \left(-\hat{\jmath} \Omega_{k} \sin \theta_{11}\right) & \cdots & \exp \left(-\hat{\jmath} \Omega_{k} \sin \theta_{l M_{1}}\right) & \cdots & \exp \left(-\hat{\jmath} \Omega_{k} \sin \theta_{L M_{L}}\right) \\
\vdots & \ddots & \vdots & \ddots & \vdots \\
\underbrace{\exp \left(-\hat{\jmath} \Omega_{P-1} \sin \theta_{11}\right)}_{\boldsymbol{a}\left(\boldsymbol{\theta}_{(1,1)}\right)} & \cdots & \underbrace{\exp \left(-\hat{\jmath} \Omega_{P-1} \sin \theta_{l M_{L}}\right)}_{\boldsymbol{a}\left(\boldsymbol{\theta}_{\left(l, M_{l}\right)}\right)} & \cdots & \underbrace{\exp \left(-\hat{\jmath} \Omega_{P-1} \sin \theta_{L M_{L}}\right)}_{\boldsymbol{a}\left(\boldsymbol{\theta}_{\left(L, M_{L}\right)}\right)}
\end{array}\right] \text { with } \Omega_{k}=\frac{k \omega d}{c} .
$$




\section{TR LOCALIZATION}

This section presents the TR source localization (DOA and range estimation) algorithms applied to an active array (passive target) setup. The TR/DOA estimation is based on the wideband Capon estimation algorithm, while the TR range estimation is based on the TR maximum likelihood algorithm. For a static target, parameter $\boldsymbol{\alpha}$ is a vector containing the range $\left(R_{l}\right)$ and DOA $\left(\theta_{l}\right)$ of the targets, and is defined as follows:

$$
\boldsymbol{\alpha}=\left[\alpha_{1}, \ldots, \alpha_{2 L}\right]^{T}=\left[R_{1}, \theta_{1}, \ldots, R_{L}, \theta_{L}\right]^{T} .
$$

Our goal is to present two TR based algorithms: one for estimating DOA $\theta_{l}$ of target $l$ buried in rich multipath 2-D environment, and; the other for estimating the range $R_{l}$. The TR range estimator has been reported previously in [13] for a single pair of antenna elements. Here, we briefly describe its extension to multiple element arrays for the single target case.

\section{A. TR DOA Estimation}

Before explaining the TR Capon algorithm for DOA estimation, we first review the conventional Capon algorithm.

1) Wideband Capon Algorithm: Following (8), the wideband Capon algorithm divides the frequency spectrum of the forward observations vector $\mathbf{r}_{k}\left(e^{\hat{\jmath} \omega}\right)$ into $Q$ frequency bins. The value of $Q$ is bounded by the signal bandwidth (BW) and coherence bandwidth $B_{c}$ of the channel to $Q_{0}=\mathrm{BW} / B_{c}$. A choice of $Q>Q_{0}$ leads to an oversampled channel and is preferable. See [9] for further details. Denoting the frequency component of the observation in the $q$ th bin as $\mathbf{r}_{k}\left(e^{\hat{\jmath} \omega_{q}}\right)$, the corresponding $q$ th bin spatial covariance matrix is given by

$$
\begin{aligned}
\mathbf{R}_{\mathbf{r}}\left(e^{\hat{\jmath} \omega_{q}}\right) & =\mathbb{E}\left\{\mathbf{r}_{k}\left(e^{\hat{\jmath} \omega_{q}}\right) \mathbf{r}_{k}^{H}\left(e^{\hat{\jmath} \omega_{q}}\right)\right\} \\
& \approx \frac{1}{N} \sum_{m=1}^{N} \mathbf{r}_{k, m}\left(e^{\hat{\jmath} \omega_{q}}\right) \mathbf{r}_{k, m}^{H}\left(e^{\hat{\jmath} \omega_{q}}\right)
\end{aligned}
$$

where $\mathbb{E}$ denotes the expectation operator. As shown in the second expression in (21), the spatial covariance matrix is estimated from the $N$ snapshots of the observation vector $\mathbf{r}_{k}\left(e^{\hat{\jmath} \omega_{q}}\right)$ within bin $q$. The Capon algorithm is traditionally expressed as the minimization problem

$$
\begin{aligned}
\min _{\mathbf{w}} & \mathbf{w}^{H}\left(e^{\hat{\jmath} \omega_{q}}, \theta\right) \mathbf{R}_{\mathbf{r}}\left(e^{\hat{\jmath} \omega_{q}}\right) \mathbf{w}\left(e^{\hat{\jmath} \omega_{q}}, \theta\right) \\
\text { subject to } & \mathbf{w}^{H}\left(e^{\hat{\jmath} \omega_{q}}, \theta\right) \boldsymbol{a}\left(e^{\hat{\jmath} \omega_{q}}, \theta\right)=1 .
\end{aligned}
$$

In other words, the Capon algorithm attempts to minimize the power contributed by noise and other interference signals while maintaining a fixed gain in the direction from which the signal originated. In our case, this direction is the direct path DOA $\theta_{(l, 1)}$ for target $l$. As defined previously in (9), the steering vector $\boldsymbol{a}\left(e^{\hat{\jmath} \omega_{q}}, \theta\right)$ is given by

$\boldsymbol{a}\left(e^{\hat{\jmath} \omega_{q}}, \theta\right)=\left[1, \exp \left(-\hat{\jmath} \Omega_{1} \sin \theta\right), \ldots, \exp \left(-\hat{\jmath} \Omega_{P-1} \sin \theta\right)\right]^{T}$

with $\Omega_{k}=k \omega d / c$. The solution to (22) is given by

$$
\mathbf{w}\left(e^{\hat{\jmath} \omega_{q}}, \theta\right)=\frac{\mathbf{R}_{\mathbf{r}}^{-1}\left(e^{\hat{\jmath} \omega_{q}}\right) \boldsymbol{a}\left(e^{\hat{\jmath} \omega_{q}}, \theta\right)}{\boldsymbol{a}^{H}\left(e^{\hat{\jmath} \omega_{q}}, \theta\right) \mathbf{R}_{\mathbf{r}}^{-1}\left(e^{\hat{\jmath} \omega_{q}}\right) \boldsymbol{a}\left(e^{\hat{\jmath} \omega_{q}}, \theta\right)}
$$

where $\theta$ is the search angle. Substituting $\mathbf{w}\left(e^{\hat{\jmath} \omega_{q}}, \theta\right)$ in expression (22), the narrowband power spectrum is given by

$$
\mathbb{P}\left(e^{\hat{\jmath} \omega_{q}}, \theta\right)=\frac{1}{\boldsymbol{a}^{H}\left(e^{\hat{\jmath} \omega_{q}}, \theta\right) \mathbf{R}_{\mathbf{r}}^{-1}\left(e^{\hat{\jmath} \omega_{q}}\right) \boldsymbol{a}\left(e^{\hat{\jmath} \omega_{q}}, \theta\right)} .
$$

Combining results from different bins, the geometrically averaged wideband capon has the power spectrum

$$
\mathbb{Q}(\theta)=\prod_{q=1}^{Q} \frac{1}{\boldsymbol{a}^{H}\left(e^{\hat{\jmath} \omega_{q}}, \theta\right) \mathbf{R}_{\mathbf{r}}^{-1}\left(e^{\hat{\jmath} \omega_{q}}\right) \boldsymbol{a}\left(e^{\hat{\jmath} \omega_{q}}, \theta\right)} .
$$

The Capon algorithm plots $\mathbb{Q}(\theta)$ as a function of $\theta$ and determines the DOAs by selecting the values of $\theta$ corresponding to $L$ highest peaks in spectrum $\mathbb{Q}(\theta)$.

2) TR Wideband Capon Algorithm: Following (15), we apply the same procedure as in the conventional Capon to the TR observations $\mathbf{p}_{k}\left(e^{\hat{\jmath} \omega_{q}}\right)$. Denoting the TR spatial covariance matrix in the $q$ th frequency bin as

$$
\begin{aligned}
\mathbf{R}_{\mathbf{p}}\left(e^{\hat{\jmath} \omega_{q}}\right) & =\mathbb{E}\left\{\mathbf{p}_{k}\left(e^{\hat{\jmath} \omega_{q}}\right) \mathbf{p}_{k}^{H}\left(e^{\hat{\jmath} \omega_{q}}\right)\right\} \\
& \approx \frac{1}{N} \sum_{m=1}^{N} \mathbf{p}_{k, m}\left(e^{\hat{\jmath} \omega_{q}}\right) \mathbf{p}_{k, m}^{H}\left(e^{\hat{\jmath} \omega_{q}}\right)
\end{aligned}
$$

the TR Capon takes the form

$$
\begin{array}{rc}
\min _{\mathbf{\mathrm { TR } _ { \mathrm { R } }}} & \mathbf{w}_{\mathrm{TR}}^{H}\left(e^{\hat{\jmath} \omega_{q}}, \theta\right) \mathbf{R}_{\mathbf{p}}\left(e^{\hat{\jmath} \omega_{q}}\right) \mathbf{w}_{\mathrm{TR}}\left(e^{\hat{\jmath} \omega_{q}}, \theta\right) \\
\text { subject to } & \mathbf{w}_{\mathrm{TR}}^{H}\left(e^{\hat{\jmath} \omega_{q}}, \theta\right) \boldsymbol{a}_{\mathrm{TR}}\left(e^{\hat{\jmath} \omega_{q}}, \theta\right)=1
\end{array}
$$

in which $a_{\mathrm{TR}}(\theta)$ is the steering vector for the TR step. The solution to the TR minimization problem is similar to the conventional approach with the TR steering vector $\boldsymbol{a}_{\mathrm{TR}}\left(e^{\hat{\jmath} \omega_{q}}, \theta\right)=$ $\boldsymbol{a}\left(e^{\hat{\jmath} \omega_{q}}, \theta\right)$ and the weight vector given by

$$
\mathbf{w}_{\mathrm{TR}}\left(e^{\hat{\jmath} \omega_{q}}, \theta\right)=\frac{\mathbf{R}_{\mathbf{p}}^{-1}\left(e^{\hat{\jmath} \omega_{q}}\right) \boldsymbol{a}\left(e^{\hat{\jmath} \omega_{q}}, \theta\right)}{\boldsymbol{a}^{H}\left(e^{\hat{\jmath} \omega_{q}}, \theta\right) \mathbf{R}_{\mathbf{p}}^{-1}\left(e^{\hat{\jmath} \omega_{q}}\right) \boldsymbol{a}\left(e^{\hat{\jmath} \omega_{q}}, \theta\right)} .
$$

In other words, the TR Capon algorithm follows the same approach as the conventional Capon except that the TR observations $\mathbf{p}_{k}\left(e^{\hat{\jmath} \omega}\right)$ are used instead of $\mathbf{r}_{k}\left(e^{\hat{\jmath} \omega}\right)$. The DOA estimates are obtained from the $L$ peaks of the TR spectrum

$$
\mathbb{Q}_{\mathrm{TR}}(\theta)=\prod_{q=1}^{Q} \frac{1}{\boldsymbol{a}^{H}\left(e^{\hat{\jmath} \omega_{q}}, \theta\right) \mathbf{R}_{\mathbf{p}}^{-1}\left(e^{\hat{\jmath} \omega_{q}}\right) \boldsymbol{a}\left(e^{\hat{\jmath} \omega_{q}}, \theta\right)}
$$

obtained by combining the results for $Q$-frequency bins.

Substituting the expression for the forward observation vector $\mathbf{r}_{k}\left(e^{\hat{\jmath} \omega}\right)$ from (8) in (16), we have

$$
\begin{aligned}
\mathbf{p}_{k}\left(e^{\hat{\jmath} \omega}\right)= & \underbrace{\mathbf{A}(\boldsymbol{\Theta}) \boldsymbol{X} \boldsymbol{\Upsilon}\left(e^{\hat{\jmath} \omega}\right) g}_{\boldsymbol{B}\left(e^{\left.\hat{\jmath} \omega_{q}, \theta\right)}\right.} \\
& \times \mathbf{A}^{*}(\boldsymbol{\Theta}) \boldsymbol{X}^{*} \boldsymbol{\Gamma}_{k}^{*}\left(e^{\hat{\jmath} \omega}\right) F^{*}\left(e^{\hat{\jmath} \omega}\right)+\mathbf{w}_{k}\left(e^{\hat{\jmath} \omega}\right) .
\end{aligned}
$$

Ignoring the noise component, we note

$$
\boldsymbol{p}_{k}\left(e^{\hat{\jmath} \omega_{q}}\right)=\boldsymbol{B}\left(e^{\hat{\jmath} \omega_{q}}, \theta\right) \times \boldsymbol{r}_{k}^{*}\left(e^{\hat{\jmath} \omega_{q}}\right)
$$

In other words, the TR operation provides a mechanism similar to the beamspace beamforming used in array signal processing, 
which focuses the beam towards the DOA $\theta_{l}$ using multipath to its advantage. Therefore, we expect more accurate DOA estimation using this approach.

\section{B. TR Range Estimation}

Since the focus of the paper is primarily on direction finding, we present the TR range estimation algorithm briefly for the sake of completeness. The range estimator is presented in terms of a single target embedded in a multipath environment. Extension to multiple targets will be made in a future work.

1) Conventional Range Estimation: For clarification, we first present the conventional range estimation algorithm for a pair of antenna arrays based on the conventional observations, (5), and then present our TR range estimation algorithm. Since the range is typically expressed in terms of round trip delay time of the probing signal to and back from the target, conventional range estimation is equivalent to estimating $\tau_{(l, i, k, j)}$ associated with the direct path $(i=1)$ from target $l$ with element $k$ probing and element $j$ receiving. Assuming that a single target $(l=1)$ is present, the time delay $\tau_{(l, i, k, j)}=\tau_{(1,1, k, j)}$ is expressed in terms of two components: i) Reference delay $\tau_{(1,1, k, 1)}$ associated with element 1 of the receiving array, and ii) Interelement delay $\Delta \tau_{(1,1, j)}$ between reference receiving element $(j=1)$ and other elements $j$ based on the direction of arrival $\theta_{l}$. The first step in the conventional range estimator is to estimate the delay for the direct path $\tau_{(1,1, k, j)}$ for each of receiving array elements with respect to target $(l=1)$ when element $k$ is probing the channel and element $j$ is receiving. For this purpose, the ML algorithm based on the conventional observations $r_{(k, j)}(t)(5)$, for $(1 \leq j \leq P)$, is used, i.e.,

$$
\begin{aligned}
& \forall(1 \leq j \leq P), \\
& \quad \hat{\tau}_{(1,1, k, j)}=\underset{\tau}{\arg \max } \Re\left\{\int r_{(k, j)}(t) f^{*}(t-\tau) d t\right\}
\end{aligned}
$$

which is equivalent to applying a matched filter with impulse response $h_{\mathrm{MF}}(t)=f^{*}(-t)$ with the forward probing signal $f(t)$. The ML algorithm assumes that the multipath attenuation (alternatively, the signal loss) is higher than that of the direct path. The second step corrects for the interelement delay $\Delta \tau_{(1,1, j)}$ in $\hat{\tau}_{(1,1, k, j)}$ for $(j \neq 1)$ and combines the $P$ estimates to determine the delay with respect to the reference receiving element $(j=1)$. With the array geometry known, the corrections can be calculated by using the estimated value $\hat{\theta}_{l}$ of the DOA. The mean of the corrected estimates $\hat{\tau}_{(1,1, k, 1)}$ then provides the overall delay estimate with respect to the transmit element $k$ and reference receive element $j=1$. A similar approach based only on a direct path propagation model has been reported in [28], which also uses antenna arrays but with widely separated elements for range estimation. Consequently, the attenuation factors and the corresponding SNRs for the setup used in [28] are different at each antenna element even for the backscatter received via the same path and reflected from the same target. The overall estimate for $\tau_{(1,1, k, 1)}$ in [28] is then obtained as a weighted average of the individual delay estimates obtained at each receiver element with the weights proportional to the SNR estimates at the respective elements. For colocated antennas, we take advantage of the DOA information to come up with more accurate estimates for the correction terms.

2) TR Range Estimator: The TR range estimator follows an approach similar to the conventional range estimator except that the TR observation vector $\mathbf{p}_{k}\left(e^{\hat{\jmath} \omega}\right)$, as given in (15), is used, which is correlated element-by-element with the TR probing signal vector, i.e.,

$$
\begin{aligned}
\forall(1 \leq & j \leq P), \hat{\tau}_{(1,1, k, j)}^{\mathrm{TR}} \\
& =\underset{\tau}{\arg \max } \Re\left\{\int\left\{\mathbf{p}_{k}(t)\right\}_{(j)}\left\{\mathbf{z}^{*}(t-\tau)\right\}_{(j)} d t\right\}
\end{aligned}
$$

where $\left\{\mathbf{p}_{k}(t)\right\}_{(j)}$ denotes the $j$ th entry of $\mathbf{p}_{k}(t)$, the later corresponding to the inverse Fourier transform of the TR observation vector $\mathbf{p}_{k}\left(e^{\hat{\jmath} \omega}\right)$. Similarly, $\{\mathbf{z}(t)\}_{(j)}$ denotes the $j$ th entry of $\mathbf{z}(t)$, the inverse Fourier transform of the probing vector $\mathbf{z}\left(e^{\hat{\jmath} \omega}\right)=g \mathbf{r}_{k}^{*}\left(e^{\hat{\jmath} \omega}\right)$ used in the TR stage. Since the observations $\mathbf{r}_{k}\left(e^{\hat{\jmath} \omega}\right)$ made in the forward probing stage are noisy, the average of several snapshots may instead be used to determine $\mathbf{z}\left(e^{\hat{\jmath} \omega}\right)$. As for the conventional range estimator, (33) results in $P$ estimates of $\hat{\tau}_{(1,1, k, j)}^{\mathrm{TR}}$. The second step uses the estimated value $\hat{\theta}_{l}$ obtained from the TR/DOA estimation algorithm that is shown to be more accurate than the conventional DOA estimation algorithm to correct for the interelement delays $\Delta \tau_{(1,1, j)}$ in $\hat{\tau}_{(1,1, k, j)}^{\mathrm{TR}}$ and combines the corrected $P$ estimates to determine the overall delay estimate with respect to the reference receiving element $(j=1)$ by taking the mean of the individual estimates.

\section{CRAMÉR-RAO LOWER BOUNDS}

In this section, we derive the CRBs for both the conventional and TR DOA/range estimators for $L$ passive targets embedded in a multipath environment based on the frequency representation (3) of the FIM of the unknown vector $\boldsymbol{\alpha}$.

\section{A. Analytical Expressions for CRBs}

Theorem 1 derives a lower bound on the accuracy of the conventional DOA and range estimators based on (13). Likewise, Theorem 2 expresses the lower bound on the accuracy of the TR DOA and range estimators based on (18). The proofs of the two theorems are included in the Appendix at the end of the paper.

Theorem 1: The CRB for the direction of arrival and the range of the targets based on the forward observation vector $\mathbf{r}_{k}\left(e^{\hat{\jmath} \omega}\right)$ (13) for the conventional estimator is given by

$$
\mathrm{CRB}^{(\mathrm{CV})}(\boldsymbol{\alpha})^{-1}=\frac{N}{\pi \sigma_{v}^{2}} \Re\left\{\int\left|F\left(e^{\hat{\jmath} \omega}\right)\right|^{2} \mathbf{D}^{H} \mathbf{D} d \omega\right\}
$$

where the $(P \times 2 L)$ derivative matrix $\mathbf{D}$ is

$$
\mathbf{D}=\left[\frac{\partial \mathbf{h}_{k}}{\partial R_{1}} \frac{\partial \mathbf{h}_{k}}{\partial \theta_{1}} \cdots \frac{\partial \mathbf{h}_{k}}{\partial R_{L}} \frac{\partial \mathbf{h}_{k}}{\partial \theta_{L}}\right]
$$

and the vector $\mathbf{h}_{k}=\mathbf{H}\left(e^{\hat{\jmath} \omega}\right) \mathbf{e}_{k}$ is the channel response vector in the forward probing stage. The matrix $\mathbf{D}$ groups all derivatives of the channel response vector $\mathbf{h}_{k}$ with respect to the source 
location parameters $R_{l}$ and $\theta_{l}$. Based on (14), the partial derivatives of the channel response matrix in (35) can be represented as

$$
\frac{\partial \mathbf{h}_{k}}{\partial R_{l}}=\boldsymbol{A}(\boldsymbol{\Theta}) \boldsymbol{X}\left(\frac{\partial \boldsymbol{\Gamma}_{k}\left(e^{\hat{\omega} \omega}\right)}{\partial R_{l}}\right)
$$

and

$$
\frac{\partial \mathbf{h}_{k}}{\partial \theta_{l}}=\left(\frac{\partial \boldsymbol{A}(\boldsymbol{\Theta})}{\partial \theta_{l}}\right) \boldsymbol{X} \boldsymbol{\Gamma}_{k}\left(e^{\hat{\jmath} \omega}\right) .
$$

As a special case, we consider the conventional range estimator based on a 2-multipath model $\left(M_{1}=2\right)$, a single pair of transmitter and receiver $(P=1)$, and a single target $(L=1)$ in Corollary 1.1. For this limited configuration $\boldsymbol{\alpha}=R_{1}$ and the setup is incapable of determining the DOA, hence, the CRB for DOA is not considered here. Note that Corollary 1.1 has been presented before in [13]. Our intent here is to illustrate that Corollary 1.1 is a special case of Theorem 1 as such the derivation of Corollary 1.1 (included in the Appendix of the manuscript) is based on (34).

Corollary 1.1: The CRLB for the conventional range (delay) estimator for a 2-multipath model considering a single target and a single pair of transmitter and receiver is given by (38), shown at the bottom of the page, where $\tau_{(1,1)}=\tau$ and $\tau_{(1,2)}=$ $\tau+\Delta \tau$. Variables $\beta_{0}^{2}$ and $\beta_{\Delta \tau}^{2}$ are given by

$$
\beta_{0}^{2}=\int \omega^{2}\left|F\left(e^{j \omega}\right)\right|^{2} d \omega
$$

and

$$
\beta_{\Delta \tau}^{2}=\int \omega^{2} \cos (\omega \Delta \tau)\left|F\left(e^{j \omega}\right)\right|^{2} d \omega .
$$

Corollary 1.1 expresses the CRB for the round-trip time delay $\tau$ for the probing waveform to travel out to the target from the transmitter and back to the receiver. Expressed in terms of the root mean square error (RMSE) $\sigma_{\tau}$ for the time delay $\tau$, the RMSE error $\sigma_{R_{1}}$ for the range (for an unbiased estimator) is given by $\sigma_{R_{1}}=\left(c_{o} / 2\right) \sigma_{\tau}$, where $c_{o}$ is the propagation velocity. Finally, we note that Corollary 1.1 is similar to the result presented in [29] for a single target time-delay estimator based on the 2-multipath model for a communication system. Theorem 1 is much more general covering both range and DOA estimators for any order of multipath.

Next, we consider the CRB for the TR DOA and range estimators.

Theorem 2: The CRB of the DOA and range of the targets based on the TR observation vector $\mathbf{p}_{k}\left(e^{\hat{\jmath} \omega}\right)$ (18) for the TR estimators is given by

$$
\mathrm{CRB}^{(\mathrm{TR})}(\boldsymbol{\alpha})^{-1}=\frac{N g^{2}}{\pi \sigma_{w}^{2}} \Re\left\{\int\left|F\left(e^{\hat{\jmath} \omega}\right)\right|^{2} \mathbf{E}^{H} \mathbf{E} d \omega\right\}
$$

where the $(P \times 2 L)$ derivative matrix $\mathbf{E}$ is given by

$$
\mathbf{E}=\left[\frac{\partial \mathbf{t}_{k}}{\partial R_{1}} \frac{\partial \mathbf{t}_{k}}{\partial \theta_{1}} \cdots \frac{\partial \mathbf{t}_{k}}{\partial R_{L}} \frac{\partial \mathbf{t}_{k}}{\partial \theta_{L}}\right]
$$

and vector $\mathbf{t}_{k}=\mathbf{T}\left(e^{\hat{j} \omega}\right) \mathbf{e}_{k}$ is the $k$ th column of the TR matrix $\mathbf{T}\left(e^{\hat{\jmath} \omega}\right)=\mathbf{H}\left(e^{\hat{\jmath} \omega}\right) \mathbf{H}^{*}\left(e^{\hat{\jmath} \omega}\right)$, which represents the channel response in the TR stage. The matrix $\mathbf{E}$ groups all the derivatives of the time reversal channel response vector $\mathbf{t}_{k}$ with respect to the source location parameters $R_{l}$ and $\theta_{l}$.

To show that the CRB presented for the TR range estimator in [13] is a special case of Theorem 2, we consider the 2-multipath model with $M_{1}=2$, and a single pair of transmitter and receiver $P=1$, and a single target $L=1$ as used in [13]. For such a configuration, $\boldsymbol{\alpha}=R_{1}$ and Theorem 2 reduces to the following form. The proof of Corollary 2.1 from Theorem 2 is included in the Appendix of the manuscript.

Corollary 2.1: The CRLB for the TR range (delay) estimator for a 2-multipath model considering a single target and a single pair of transmitter and receiver is given by

$$
\begin{aligned}
\operatorname{var} & \left\{\sigma_{\hat{\tau}}^{(\mathrm{TR})}\right\} \geq\left(\pi \sigma_{w}^{2}\right) /\left(g^{2} N\right) \\
& \times\left\{\left[\left(\left|X_{(1,1)}\right|^{4}+\left|X_{(1,2)}\right|^{4}+4\left|X_{(1,1)}\right|^{2}\left|X_{(1,2)}\right|^{2}\right) \beta_{0}^{2}\right.\right. \\
& +4 \Re\left\{X_{(1,1)}^{*} X_{(1,2)}\right\}\left(\left|X_{(1,1)}\right|^{2}+\left|X_{(1,2)}\right|^{2}\right) \beta_{\Delta \tau}^{2} \\
& \left.\left.+2 \Re\left\{\left(X_{(1,1)}^{*} X_{(1,1)}\right)^{2}\right\} \beta_{2 \Delta \tau}^{2}\right]\right\}^{-1}
\end{aligned}
$$

where $\beta_{2 \Delta \tau}^{2}$ is given by

$$
\beta_{2 \Delta \tau}^{2}=\int \omega^{2} \cos (2 \omega \Delta \tau)\left|F\left(e^{\hat{\jmath} \omega}\right)\right|^{2} d \omega .
$$

As in Corollary 1.1, Corollary 2.1 derives the CRB for the round-trip delay $\tau$. Again, the CRB for the range can be determined from the equality $\sigma_{R_{1}}=\left(c_{o} / 2\right) \sigma_{\tau}$. Finally, we note that Corollary 2.1 is similar to the result proved in [29] for the single target TR delay estimator based on the 2-multipath model in a communication system. As for Theorem 1, Theorem 2 is much more general and covers the CRBs for both TR range and DOA estimators for any order of multipath and any number of targets.

\section{B. Analytical Interpretation of the CRBs}

In this section, we analyze the contributions of the multipath to both the conventional and TR CRBs. Our goal is to express $\mathbf{D}^{H} \mathbf{D}$ and $\mathbf{E}^{H} \mathbf{E}$ in terms of known parameters so that the corresponding CRBs can be plotted. For simplicity, we restrict our analysis to a single target $(L=1)$ though the results are generalizable to $L$ targets. For a single target, the $(P \times 2)$ conventional Jacobian matrix $\mathbf{D}=\left[H_{1 k}^{\bullet}, \ldots, H_{P k}^{\bullet}\right]^{T}$, where

$$
\operatorname{var}\left\{\sigma_{\hat{\tau}}^{(\mathrm{CV})}\right\} \geq \frac{\pi \sigma_{v}^{2}}{N\left[\left(\left|X_{(1,1)}\right|^{2}+\left|X_{(1,2)}\right|^{2}\right) \beta_{0}^{2}+2 \Re\left\{X_{(1,1)} X_{(1,2)}^{*}\right\} \beta_{\Delta \tau}^{2}\right]},
$$


$H_{j k}^{\bullet}=\partial H_{j k} / \partial \boldsymbol{\alpha}$ is a $(2 \times 1)$ vector. Term $\mathbf{D}^{H} \mathbf{D}$, therefore, reduces to

$$
\mathbf{D}^{H} \mathbf{D}=\left[\begin{array}{cc}
\sum_{j=1}^{P}\left|\frac{\partial H_{j k}}{\partial R}\right|^{2} & \sum_{j=1}^{P} \frac{\partial H_{j k}^{*}}{\partial R} \frac{\partial H_{j k}}{\partial \theta} \\
\sum_{j=1}^{P} \frac{\partial H_{j k}^{*}}{\partial \theta} \frac{\partial H_{j k}}{\partial R} & \sum_{j=1}^{P}\left|\frac{\partial H_{j k}}{\partial \theta}\right|^{2}
\end{array}\right] .
$$

Similarly, for a single target, the $(P \times 2)$ TR Jacobian matrix $\mathbf{E}=\left[T_{1 k}^{\bullet}, \ldots, T_{P k}^{\bullet}\right]^{T}$ with $T_{j k}^{\bullet}=\partial T_{j k} / \partial \boldsymbol{\alpha}$ and, therefore, term $\mathbf{E}^{H} \mathbf{E}$ reduces to

$$
\mathbf{E}^{H} \mathbf{E}=\left[\begin{array}{cc}
\sum_{j=1}^{P}\left|\frac{\partial T_{j k}}{\partial R}\right|^{2} & \sum_{j=1}^{P} \frac{\partial T_{j k}^{*}}{\partial R} \frac{\partial T_{j k}}{\partial \theta} \\
\sum_{j=1}^{P} \frac{\partial T_{j k}^{*}}{\partial \theta} \frac{\partial T_{j k}}{\partial R} & \sum_{j=1}^{P}\left|\frac{\partial T_{j k}}{\partial \theta}\right|^{2}
\end{array}\right] .
$$

Expressions (45) and (46) are used to derive the CRBs in the experimental simulations considered next. Note that the channel response matrix $\mathbf{H}$ and TR matrix $\mathbf{T}$ are determined experimentally by sending a probing signal sequentially from each element in the antenna array and recording the backscatters. The TR matrix incorporates the additional TR probing step. The process may be repeated and an average of the backscatter observations is used to reduce the impact of noise.

\section{EXPERIMENTAL SIMULATIONS}

In this section, we investigate the performance of the TR/DOA estimator and compare it with the conventional DOA estimator. The implementations of the DOA estimators are based on the Capon algorithm and are described in Section IV-A. Our comparisons are based on the following four characteristics: i) root mean square errors (RMSE) of the estimators based on the Monte Carlo simulations; ii) resolution capability; iii) ability to accurately estimate the DOA of a target in the presence of a nearby multipath; and iv) the CRBs illustrating the potential performance possible with the two approaches. For simplicity of implementation, we assume a uniform linear array compromising of $P=10$ sensors with interelement sensor spacing $d$ set to $\lambda_{\min } / 2$, where $\lambda_{\min }$ is the minimum wavelength present in the wideband signal. The probing signal is taken to be a pulse with linear frequency modulation (LFM), $\left(f(t)=\tilde{f}(t) e^{\hat{\jmath} \omega_{0} t}\right)$, where the angular frequency $\omega_{0}=2 \pi f_{0}$ and the base chirp frequency $f_{0}$ equals $5 \mathrm{GHz}$. The complex envelope $\tilde{f}(t)$ of the probing signal is

$$
\tilde{f}(t)=\frac{1}{\sqrt{\tau}_{0}} \operatorname{Rect}\left(\frac{t}{\tau_{0}}\right) e^{\hat{\jmath} \pi \mu t^{2}} .
$$

A LFM signal such as $f(t)=\tilde{f}(t) e^{\hat{\jmath} \omega_{0} t}[10]$, [30] with $\tilde{f}(t)$ defined above is commonly used as a probing signal in most modern radar systems. By sweeping the frequency linearly across the pulse width, $f(t)$ achieves much wider operating bandwidth as shown in Fig. 2 and, therefore, a better compression gain. Both features are highly desirable for improved range/DOA resolution in radar applications. See [30] for more details. The number $Q$ of frequency bins is set to 10 ,

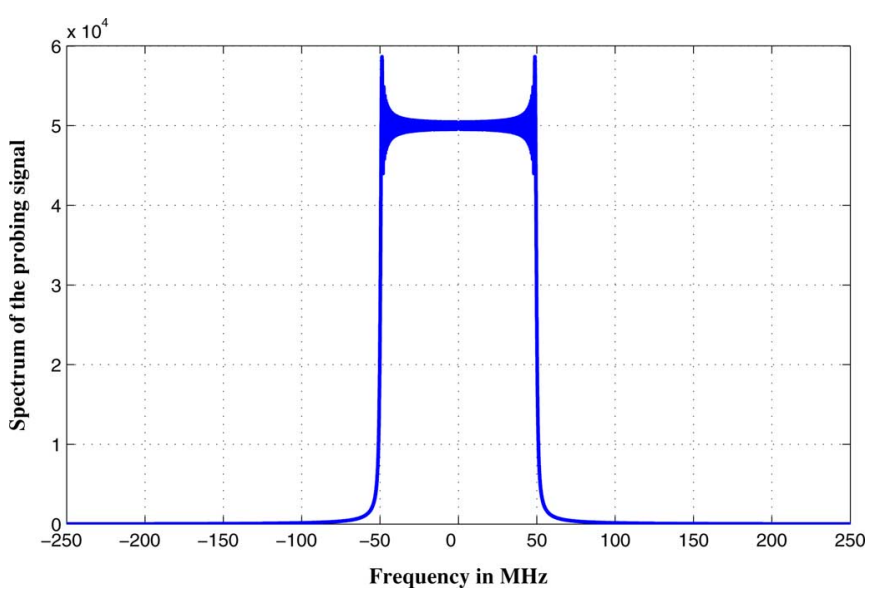

Fig. 2. Spectrum of the probing signal. The square like spectrum is widely known as the Fresnel spectrum.

a reasonable value for the 2-path, 3-path, and 4-path propagation environments discussed below. For the three setups, the pseudospectrum outputs of the conventional and TR DOA algorithms are plotted in Figs. 3 and 4, the corresponding histograms of the error distribution in Fig. 5, and the performance curves in terms of RMSE versus SNR in Fig. 6. The SNR for Figs. $3-5$ is fixed at $-15 \mathrm{~dB}$. Table II lists the estimated values of the DOA from the outputs of the conventional and TR DOA algorithms plotted in Fig. 3.

a) 2-Path Setup: The setup used in the first Monte Carlo simulation is based on a radar system shown in Fig. 1 with the constituent parameters defined in Table I. For the conventional Capon, (5) is used to model the 2-path received signals $r_{(k, j)}(t)$ with DOAs set to $\left\{40^{\circ},-30^{\circ}\right\}$ and the corresponding attenuation factors are given by $\{1, \sqrt{0.5}\}$. In terms of our notation, $X_{(1,1)}=1$ and $X_{(1,2)}=\sqrt{0.5}$, which incorporates signal loss for round trip propagation from the transmitting element to the target and back to the receiving element. The values of the reference delays for the two components are $\{6.67,7\} \mu$ s. Observation noise with different variances is added to simulate a variety of SNRs. The conventional DOA estimator estimates the DOA based on the observation $r_{(k, j)}(t)$. The TR observation is based on (15) with same values for the DOAs and attenuation factors as were used in the conventional Capon. At a SNR of $-15 \mathrm{~dB}$, the power spectra produced by the conventional and TR DOA estimators are plotted in Fig. 3(a), where for reference we plot two vertical lines at $\left\{40^{\circ}\right.$ and $\left.-30^{\circ}\right\}$ corresponding to the actual values of the DOAs (ground truth). We note that the TR/DOA estimator produces a better result on all of the performance characteristics mentioned before. There are only two peaks present in the TR/DOA spectrum (shown by the dotted line), which corresponds to the order of the multipath in the received signal. The power spectrum for the conventional Capon shows an erroneous third peak at around $-1^{\circ}$. In addition, the TR/DOA estimator is more accurate with the highest peak in its spectrum observed at $39.9^{\circ}$, much closer to the simulated DOA of $40^{\circ}$ and with much finer resolution (smaller lobes). The conventional DOA estimator computes a value of $36.2^{\circ}$ for the DOA. A second comparison is included in Fig. 4 for DOAs 


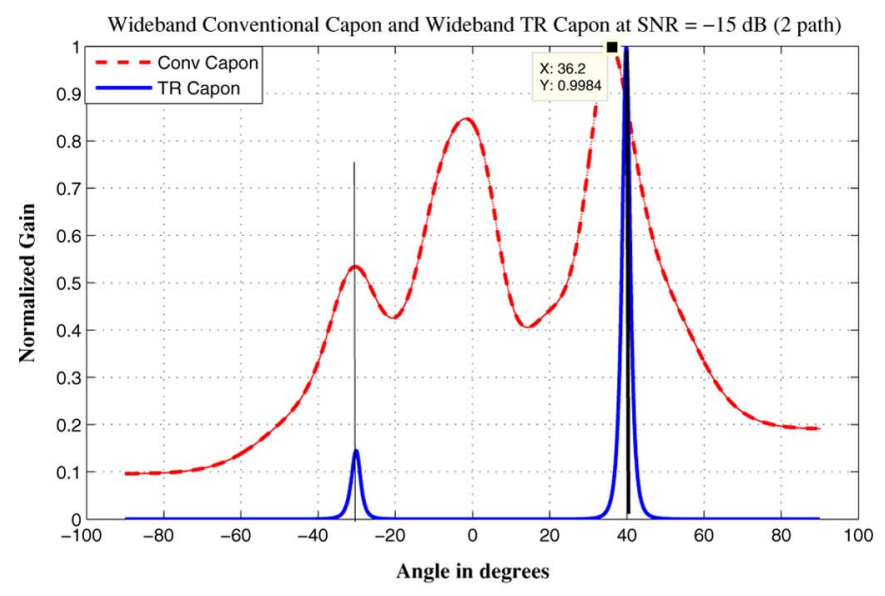

(a)

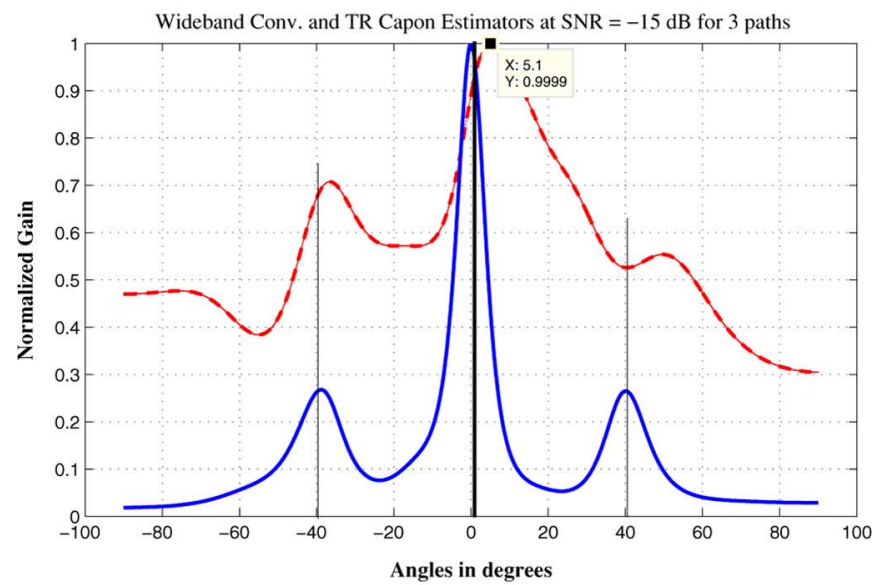

(b)

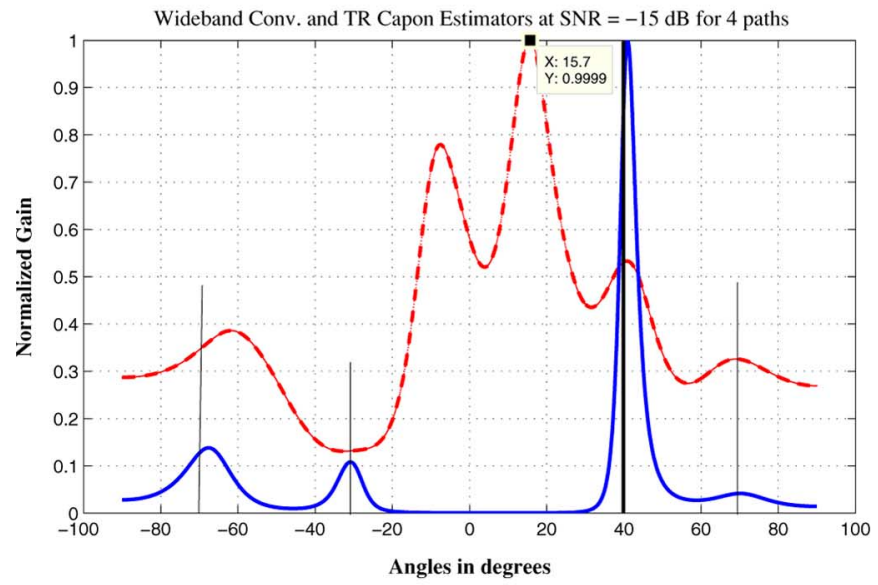

(c)

Fig. 3. Outputs of the Capon estimator based on the conventional versus TR measurements for the: (a) 2-path; (b) 3-path; and (c) 4-path propagation models.

set to $\left\{40^{\circ}, 60^{\circ}\right\}$. While the TR/DOA estimator is able to isolate the two DOAs of the waveforms originating from the target and multipath, the spectrum of the conventional DOA estimator shows only one peak illustrating that the TR approach offers superior resolution and is capable of distinguishing between approaching waveforms with fairly close DOAs.

b) 3-Path Setup: Is same as the 2-path setup except for i) DOAs set to $\left\{0^{\circ}, 40^{\circ},-40^{\circ}\right\}$; ii) attenuation factors given by

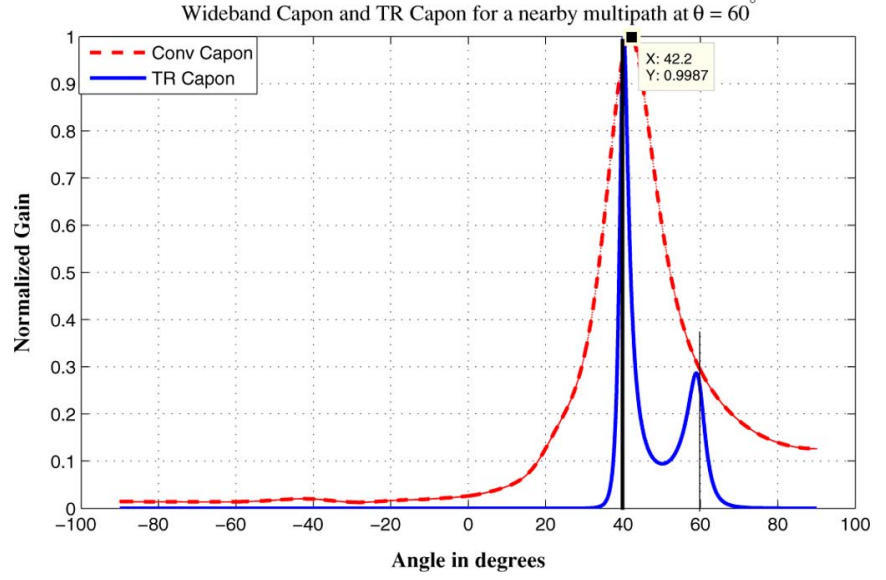

Fig. 4. Same as Fig. 3(a) except that the DOAs are set to $\left\{40^{\circ}, 60^{\circ}\right\}$.

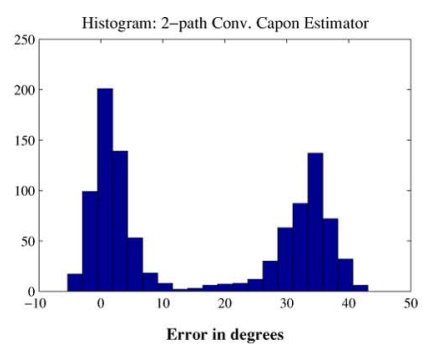

(a1)

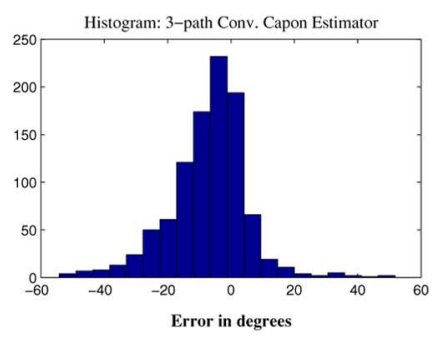

(b1)

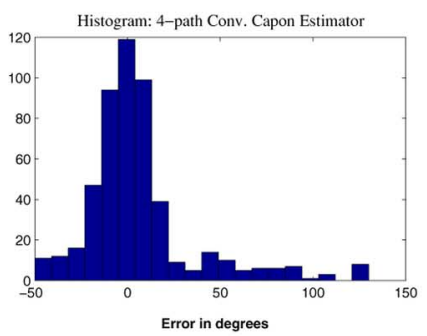

(c1)

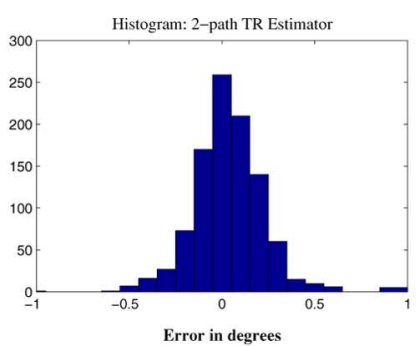

(a2)

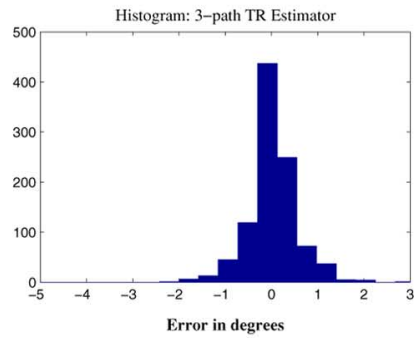

(b2)

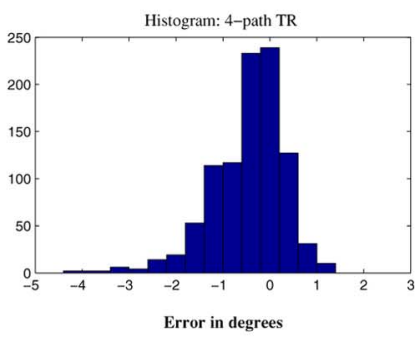

(c2)
Fig. 5. Histograms showing the error distributions for the direct path with DOAs of $40^{\circ}$ for 2 and 4 paths, and $0^{\circ}$ for 3 paths all at SNR of $-15 \mathrm{~dB}$ for the: (a1)-(a2) 2-path; (b1)-(b2) 3-path, and (c1)-(c2) 4-path environments. The histograms for the conventional Capon DOA estimator are shown on the left (a1), (b1), (c1), while the histograms for the proposed TR/DOA estimator are shown on the right (a2), (b2), (c2). Note that the scales used in the two sets of subplots are different.

$\{1, \sqrt{0.5}, \sqrt{0.5}\}$; and iii) delays of $\{6.67,7,7.33\} \mu$ s. Fig. 3(b) plots the spectrum obtained from the conventional and TR DOA algorithms at a SNR of $-15 \mathrm{~dB}$. Again, we observe that the output estimate $\left(-0.1^{\circ}\right)$ of the TR/DOA estimator is closer to the true value $\left(0^{\circ}\right)$ with a significantly narrower peak. The estimate of the conventional algorithm is $5.1^{\circ}$. 


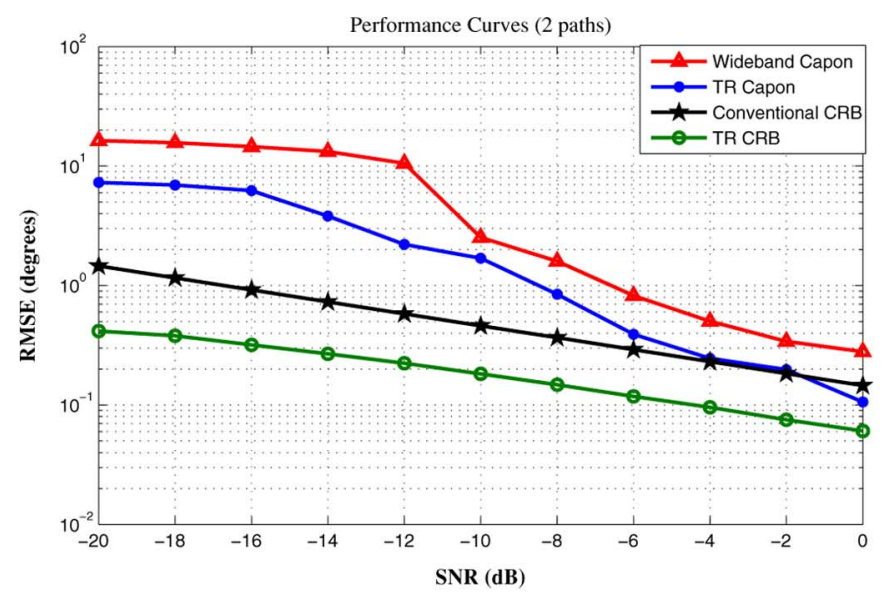

(a)

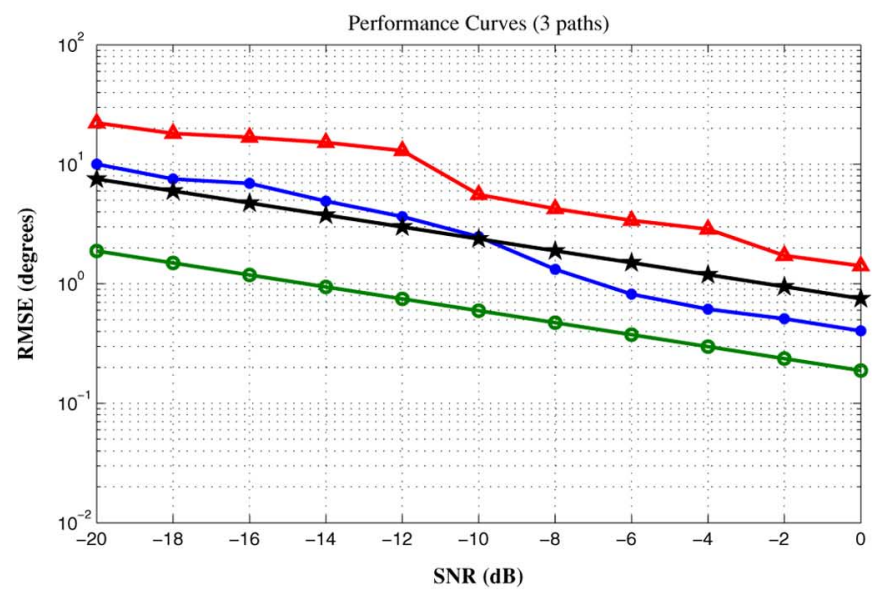

(b)

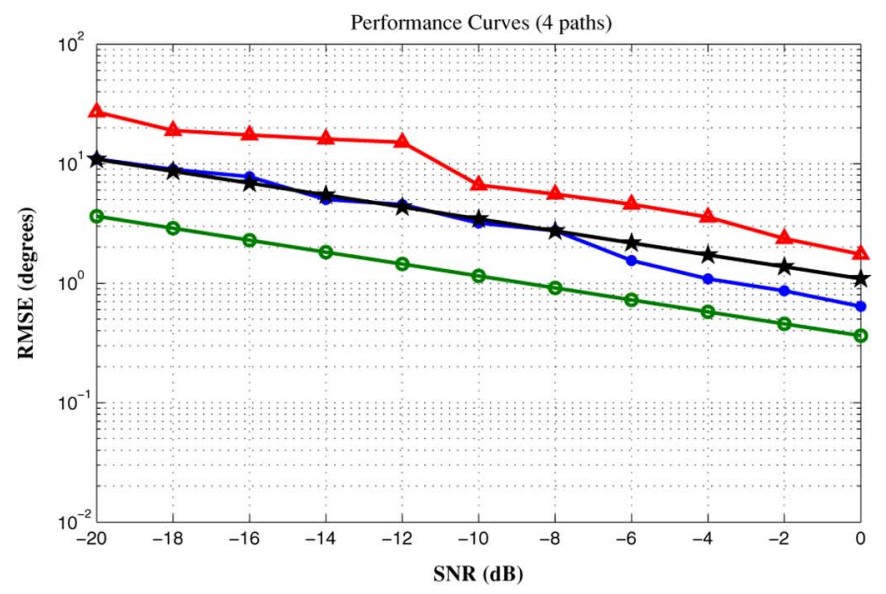

(c)

Fig. 6. Performance curves in terms of the RMSE for the conventional and TR DOA estimators for the: (a) 2-path; (b) 3-path; and (c) 4-path environment in degrees. For comparison, the plots for the CRBs are also included in the figure.

c) 4-Path Setup: Is same as Setups (a)-(b) except for i) DOAs set to $\left\{40^{\circ}, 70^{\circ},-30^{\circ},-70^{\circ}\right\}$; (ii) attenuation factors given by $\{1, \sqrt{0.5}, \sqrt{0.5}, \sqrt{0.5}\}$; and iii) delays of $\{6.66,13.3,16.0,19.3\} \mu$ s. Fig. 3(c) plots the spectrum at a SNR of $-15 \mathrm{~dB}$, which corroborates our earlier observation that the TR/DOA estimator, with the estimated DOA of $40.9^{\circ}$,
TABLE I

Parameters Used in the Monte Carlo Simulations For the 2-Path PROPAGATION SETUP

\begin{tabular}{|l|c|}
\hline Number of targets $(L)$ & 1 \\
\hline Number of multipaths $\left(M_{(l)}\right)$ & 2 \\
\hline Number of antenna elements $(P)$ & 10 \\
\hline Number of frequency bins $(Q)$ & 10 \\
\hline Direction of arrival $\left(\theta_{(l, i)}\right)$ & $\left\{40^{\circ},-30^{\circ}\right\}$ \\
\hline Time delays for the 2-path environment & $\{6.67,7\} \mu \mathrm{s}$ \\
\hline Attenuation factors & $\{1, \sqrt{0.5}\}$. \\
\hline
\end{tabular}

TABLE II

ESTIMATED DOAS FOR THE CONVENTIONAL AND TR DOA ESTIMATORS

\begin{tabular}{|l|c|c|c|}
\hline & Figure & \multicolumn{2}{|c|}{ Estimated DOA } \\
\cline { 3 - 4 } & No. & Capon & TR Capon \\
\hline 2-path $\left(\mathrm{DOA}=40^{\circ}\right)$ & $3(\mathrm{a})$ & $36.2^{\circ}$ & $39.9^{\circ}$ \\
\hline 3-path $\left(\mathrm{DOA}=0^{\circ}\right)$ & $3(\mathrm{~b})$ & $5.1^{\circ}$ & $-0.1^{\circ}$ \\
\hline 4-path $\left(\mathrm{DOA}=40^{\circ}\right)$ & $3(\mathrm{c})$ & $15.7^{\circ}$ & $40.9^{\circ}$ \\
\hline
\end{tabular}

is superior to its conventional counterpart that produces an output of $15.7^{\circ}$.

Fig. 5 plots the histograms of errors observed over 1000 simulation runs for the conventional and TR DOA estimators with respect to the main path for a SNR of $-15 \mathrm{~dB}$ for the 2-, 3-, and 4-path propagation setups described before. In all three cases, we observe that the error spread for the conventional Capon is wider than that of the TR/DOA estimator. This is a further proof of the superiority of the TR/DOA estimator over the conventional Capon. The histogram [Fig. 5(a1)] for the 2-path setup is bimodal with the second mode corresponding to the erroneous peak observed at roughly $-1^{\circ}$ in Fig. 3(a). Using Theorems 1 and 2, Fig. 6 plots the analytical CRBs for the conventional and TR DOA estimators as well as the actual performance characteristics for the two DOA estimators computed from the aforementioned numerical simulations in the 2-, 3-, and 4-path setups. The plots are expressed in terms of RMSE in degrees. The actual performance results in Fig. 6 are derived from a Monte Carlo simulation based on 1000 runs for each signal-to-noise ratio. In order to evaluate the two CRBs (conventional and TR), we derive the channel response matrix $\mathbf{H}\left(e^{\hat{\jmath} \omega}\right)$ for three different sets of values $\left\{R_{1}, \theta_{1}\right\},\left\{R_{2}, \theta_{1}\right\}$, and $\left\{R_{1}, \theta_{2}\right\}$ for the target range and DOA. For the conventional Jacobian matrix $\mathbf{D}$, we use the following centered finite difference expressions for the range and DOA derivatives

$$
\frac{\partial H_{i k}\left(e^{\hat{\jmath} \omega}\right)}{\partial R}=\frac{H_{i k}\left(e^{\hat{\jmath} \omega} ; R_{1}\right)-H_{i k}\left(e^{\hat{\jmath} \omega} ; R_{2}\right)}{\Delta R}
$$

and

$$
\frac{\partial H_{i k}\left(e^{\hat{\jmath} \omega}\right)}{\partial \theta}=\frac{H_{i k}\left(e^{\hat{\jmath} \omega} ; \theta_{1}\right)-H_{i k}\left(e^{\hat{\jmath} \omega} ; \theta_{2}\right)}{\Delta \theta}
$$

where $\Delta R=R_{1}-R_{2}$ and $\Delta \theta=\theta_{1}-\theta_{2}$. The value of $\mathbf{D}^{H} \mathbf{D}$ is then derived using (45). Likewise, for the TR/DOA CRB, we obtain $\mathbf{E}^{H} \mathbf{E}$ based on (46), where the partial derivatives for $T_{j k}\left(e^{\hat{\jmath} \omega}\right)$ are obtained by finite difference expressions similar to (48) and (49). Since the probing signal $F\left(e^{\hat{\jmath} \omega}\right), \mathbf{D}^{H} \mathbf{D}$, and $\mathbf{E}^{H} \mathbf{E}$ are defined at discrete frequencies, therefore, the CRBs are computed using the discrete implementations of (34) and (41). In field experiments, both matrices $\mathbf{H}$ and $\mathbf{T}$ are derived 
from observations recorded at the antenna array as the channel is probed sequentially by the antenna elements.

Comparing the CRBs plotted in Fig. 6, where the upper straight line drawn in black (" $\star$ ") represents the conventional CRLB and the lower straight line drawn in green ("o") represents the TR CRLB, we note that the TR/DOA estimator has a lower bound and, therefore, has a better potential of providing superior performance than the conventional DOA estimator in all three cases. Comparing the actual performance curves, we observe that the RMSE for the TR/DOA estimator (shown by curve "•") have lower values in comparison with the conventional DOA estimator (shown by curve " $\triangle$ ") especially for SNRs lower than $-5 \mathrm{~dB}$. Setting the RMSE to $2^{\circ}$, for example, in the 4-path setup, we observe that the corresponding SNR for the TR observation is about $-7 \mathrm{~dB}$ compared to around -1 $\mathrm{dB}$ for the conventional observation. For this case, this implies that the TR/DOA estimator exhibits better or the same RMSE as the conventional approach for observations with up to 6 $\mathrm{dB}$ lower SNR. Another factor to consider is the difference in the RMSEs from their CRBs at different SNRs. At $-20 \mathrm{~dB}$ in Fig. 6(c), the difference for the TR/DOA estimator is (10-3.75) or $6.25^{\circ}$, whereas for the conventional estimator is $(28-10)$ or $18^{\circ}$. At $-2 \mathrm{~dB}$, the differences decrease to about $0.35^{\circ}$ for the TR/DOA estimator and to about $1^{\circ}$ for its conventional counterpart. A further observation that we make is that RMSE curves are following the CRB but are not meeting them at SNRs in the vicinity of $0 \mathrm{~dB}$. Similar observations were made in other numerical simulations with different DOAs, multipath, or/and probing signals.

\section{SUMMARY AND FUtURE WORK}

In this paper, we introduce TR to exploit spatial/multipath diversity existing in rich scattering environments to improve the capability of target localization algorithms. We show that TR provides a built-in feature to adapt the transmitted waveform to the multipath environment and, therefore, enhances the performance of the localization algorithms. We propose TR based range and DOA estimation algorithms, and derive analytical expressions for the corresponding CRBs. Comparison of the CRBs based on the numerical simulations illustrates the potential improvement in the performance possible with the TR approaches. By exploiting multipath diversity, the TR/DOA estimator provides a mechanism for beamspace beamforming to focus the probing signal at the location of the target, which results in more accurate localization estimates.

As part of the future work, we intend to extend the proposed approaches to multiple-input multiple-output (MIMO) radar setup, where all elements in the antenna array probe the channel simultaneously using a set of orthogonal waveforms during the forward probing step. Since MIMO uses diverse signals transmitted from widely spaced antennas and provides additional spatial diversity, we expect further improvement in the accuracy of our results.

\section{APPENDIX}

Proof of Theorem 1: Based on (13), we have

$$
\mathbf{r}_{k}\left(e^{\hat{\jmath} \omega}\right)=\mathbf{h}_{k}\left(e^{\hat{\jmath} \omega}\right) F\left(e^{\hat{\jmath} \omega}\right)+\mathbf{v}_{k}\left(e^{\hat{\jmath} \omega}\right) .
$$

Therefore, the forward observations $\mathbf{r}_{k}\left(e^{\hat{\jmath} \omega}\right)$ is a $P$ dimensional complex Gaussian vector with mean $\boldsymbol{h}_{k}\left(e^{\hat{\jmath} \omega}\right) F\left(e^{\hat{\jmath} \omega}\right)$ and the power spectral density of $\mathbf{C}_{r}=\sigma_{v}^{2} \boldsymbol{I}_{p}$. Since only the mean of $\mathbf{r}_{k}\left(e^{\hat{\jmath} \omega}\right)$ is a function of the unknown parameter $\boldsymbol{\alpha}$, (3) reduces to

$$
[\mathcal{I}(\boldsymbol{\alpha})]_{i j}=\frac{N}{\pi \sigma_{v}^{2}} \Re\left\{\int \mathbf{d}_{i}^{H}\left(e^{\hat{\jmath} \omega}, \boldsymbol{\alpha}\right) \mathbf{d}_{j}\left(e^{\hat{\jmath} \omega}, \boldsymbol{\alpha}\right) d \omega\right\} .
$$

From (13), the mean value of the DTFT of $\boldsymbol{\mu}(\boldsymbol{\alpha})$ for the conventional estimator equals $\boldsymbol{h}_{k}\left(e^{\hat{\jmath} \omega}, \boldsymbol{\alpha}\right) F\left(e^{\hat{\jmath} \omega}\right)$. Substituting this mean value in (4), gives

$$
\begin{aligned}
\mathbf{d}_{i}\left(e^{\hat{\jmath} \omega}, \boldsymbol{\alpha}\right) & =\operatorname{DTFT}\left\{\frac{\partial \boldsymbol{\mu}(\boldsymbol{\alpha})}{\partial \alpha_{i}}\right\} \\
& =\frac{\partial(\operatorname{DTFT}\{\boldsymbol{\mu}(\boldsymbol{\alpha})\})}{\partial \alpha_{i}} \\
& =F\left(e^{\hat{\jmath} \omega}\right) \frac{\partial \boldsymbol{h}_{k}\left(e^{\hat{\jmath} \omega}, \boldsymbol{\alpha}\right)}{\partial \alpha_{i}} .
\end{aligned}
$$

Substituting (51) in (50), the FIM of $\boldsymbol{\alpha}$ is

$$
\begin{aligned}
& {[\mathcal{I}(\boldsymbol{\alpha})]_{i j} }=\frac{N}{\pi \sigma_{v}^{2}} \\
& \quad \times \Re\left\{\int\left|F\left(e^{\hat{\jmath} \omega}\right)\right|^{2} \frac{\partial \mathbf{h}_{k}^{H}\left(e^{\hat{\jmath} \omega}, \boldsymbol{\alpha}\right)}{\partial \alpha_{i}} \frac{\partial \mathbf{h}_{k}\left(e^{\hat{\jmath} \omega}, \boldsymbol{\alpha}\right)}{\partial \alpha_{j}} d \omega\right\} .
\end{aligned}
$$

Grouping all derivatives of vector $\mathbf{h}_{k}$ with respect to the source location parameters, $\left(R_{l}, \theta_{l}\right)$ in a $P \times 2 L$ matrix $\mathbf{D}$ [as given in (35)], proves Theorem 2.

Proof of Corollary 1.1: For range estimator and based on a 2-multipath model, $M_{(1)}=2, L=1$, and $\boldsymbol{a}(\theta)=1$. Substituting these values in (11) gives

$$
\boldsymbol{h}_{k}\left(e^{\hat{\jmath} \omega}\right)=H_{11}\left(e^{\hat{\jmath} \omega}\right)=\sum_{i=1}^{2} X_{(1, i)} \boldsymbol{a}_{j}\left(\boldsymbol{\theta}_{(1, i)}\right) e^{-j \omega \tau_{(1, i)}}
$$

With $\tau_{(1,1)}=\tau$ and $\tau_{(1,2)}=\tau+\Delta \tau$, the channel response vector is expressed as

$$
\boldsymbol{h}_{k}\left(e^{\hat{\jmath} \omega}\right)=\left[X_{(1,1)}+X_{(1,2)} e^{-\hat{\jmath} \omega \Delta \tau}\right] e^{-\hat{\jmath} \omega \tau} .
$$

The only parameter to be estimated here is the range of the target (i.e., $\alpha=R$ ), which depends on the direct path delay, $\tau$. Therefore, the derivative of the channel response vector is given by

$$
\frac{\partial \boldsymbol{h}_{k}\left(e^{\hat{\jmath} \omega}\right)}{\partial \tau}=(-\hat{\jmath} \omega)\left[X_{(1,1)}+X_{(1,2)} e^{-\hat{\jmath} \omega \Delta \tau}\right] e^{-\hat{\jmath} \omega \tau} .
$$

Consequently, the magnitude squared of the above term will be

$$
\begin{aligned}
&\left|\frac{\partial \boldsymbol{h}_{k}\left(e^{\hat{\jmath} \omega}\right)}{\partial \tau}\right|^{2}=\left[\left|X_{(1,1)}\right|^{2}+\left|X_{(1,2)}\right|^{2}\right] \omega^{2} \\
&+2 \Re\left\{X_{(1,1)} X_{(1,2)}^{*} e^{-\hat{\jmath} \omega \Delta \tau}\right\} \omega^{2} .
\end{aligned}
$$

Substituting (56) in (52), we have

$[\mathcal{I}(\boldsymbol{\alpha})]_{i j}$

$$
=\frac{N}{\pi \sigma_{v}^{2}} \int\left[\left|X_{(1,1)}\right|^{2}+\left|X_{(1,2)}\right|^{2}\right] \omega^{2}\left|F\left(e^{\hat{\jmath} \omega}\right)\right|^{2} d \omega
$$




$$
+\frac{N}{\pi \sigma_{v}^{2}} \int 2 \Re\left\{X_{(1,1)} X_{(1,2)}^{*} e^{-\hat{\jmath} \omega \Delta \tau}\right\} \omega^{2}\left|F\left(e^{\hat{\jmath} \omega}\right)\right|^{2} d \omega .
$$

The second term in (57) simplifies to

$$
\begin{aligned}
\frac{2 N}{\pi \sigma_{v}^{2}} \Re\{ & \left.X_{(1,1)} X_{(1,2)}^{*}\right\} \int \cos (\omega \Delta \tau) \omega^{2}\left|F\left(e^{\hat{\jmath} \omega}\right)\right|^{2} d \omega \\
& \quad-\frac{2 N}{\pi \sigma_{v}^{2}} \Re\left\{X_{(1,1)} X_{(1,2)}^{*}\right\} \int \sin (\omega \Delta \tau) \omega^{2}\left|F\left(e^{\hat{\jmath} \omega}\right)\right|^{2} d \omega
\end{aligned}
$$

which reduces to

$$
\frac{2 N}{\pi \sigma_{v}^{2}} \Re\left\{X_{(1,1)} X_{(1,2)}^{*}\right\} \int \cos (\omega \Delta \tau) \omega^{2}\left|F\left(e^{\hat{\jmath} \omega}\right)\right|^{2} d \omega
$$

because the function containing $\sin (\cdot)$ is an odd term and the corresponding integral reduces to 0 . Using the notations $\beta_{0}^{2}$ and $\beta_{\Delta \tau}^{2}$ as defined in (40), (57) reduces to Corollary 1.1.

Proof of Theorem 2: Based on (18), the TR observations are given by

$$
\mathbf{p}_{k}\left(e^{\hat{\jmath} \omega}\right)=g \mathbf{t}_{k}\left(e^{\hat{\jmath} \omega}\right) F^{*}\left(e^{\hat{\jmath} \omega}\right)+\mathbf{w}_{k}\left(e^{\hat{\jmath} \omega}\right)
$$

where the mean value of the DTFT of $\boldsymbol{\mu}(\boldsymbol{\alpha})$ for TR observations is $g \mathbf{t}_{k}\left(e^{\hat{\jmath} \omega}\right) F^{*}\left(e^{\hat{\jmath} \omega}\right)$ and the power spectral density of $\mathbf{p}_{k}$ is $\mathbf{C}_{p}=\sigma_{w}^{2} \boldsymbol{I}_{p}$. Since only the mean of $\mathbf{p}_{k}\left(e^{\hat{\jmath} \omega}\right)$ is a function of the unknown parameter $\boldsymbol{\alpha},(3)$ reduces to

$$
[\mathcal{I}(\boldsymbol{\alpha})]_{i j}=\frac{N}{\pi \sigma_{w}^{2}} \Re\left\{\int \mathbf{d}_{i}^{H}\left(e^{\hat{\jmath} \omega}, \boldsymbol{\alpha}\right) \mathbf{d}_{j}\left(e^{\hat{\jmath} \omega}, \boldsymbol{\alpha}\right) d \omega\right\},
$$

where

$$
\mathbf{d}_{i}\left(e^{\hat{\jmath} \omega}, \boldsymbol{\alpha}\right)=g \frac{\partial \boldsymbol{t}_{k}\left(e^{\hat{\jmath} \omega}, \boldsymbol{\alpha}\right)}{\partial \alpha_{i}} F^{*}(\omega) .
$$

Substituting the above expression in (60), we get

$$
\begin{aligned}
{[\mathcal{I}(\boldsymbol{\alpha})]_{i j} } & =\frac{N g^{2}}{\pi \sigma_{w}^{2}} \\
\times \Re & \left\{\int \frac{\partial \mathbf{t}_{k}^{H}\left(\boldsymbol{\alpha}, e^{\hat{\jmath} \omega}\right)}{\partial \alpha_{i}} \frac{\partial \mathbf{t}_{k}\left(\boldsymbol{\alpha}, e^{\hat{\jmath} \omega}\right)}{\partial \alpha_{j}}\left|F\left(e^{\hat{\jmath} \omega}\right)\right|^{2} d \omega\right\} .
\end{aligned}
$$

We group all derivatives of the vector $\mathbf{t}_{k}$ with respect to the source location parameters, $\left(R_{l}, \theta_{l}\right)$ in a $P \times 2 L$ matrix $\mathbf{E}$ [as given in (42)], which results in Theorem 2.

Proof of Corollary 2.1: Being a special case, Corollary 2.1 states that $M_{(1)}=2, L=1$, and $\boldsymbol{a}(\theta)=1$. As for Corollary 1.1, the channel response matrix (54) is given by

$$
H_{11}\left(e^{\hat{\jmath} \omega}\right)=\left[X_{(1,1)}+X_{(1,2)} e^{-\hat{\jmath} \omega \Delta \tau}\right] e^{-\hat{\jmath} \omega \tau} .
$$

Substituting (63) in $T\left(e^{\hat{\jmath} \omega}\right)=\left|H_{11}\left(e^{\hat{\jmath} \omega}\right)\right|^{2} e^{-\hat{\jmath} \omega \tau}$ gives

$$
\begin{aligned}
\boldsymbol{t}_{k}\left(e^{\hat{\jmath} \omega}\right)=T\left(e^{\hat{\jmath} \omega}\right)= & {\left[\left|X_{(1,1)}\right|^{2}+\left|X_{(1,2)}\right|^{2}\right.} \\
& \left.+2 \Re\left\{X_{(1,1)} X_{(1,2)}^{*} e^{\hat{\jmath} \omega \Delta \tau}\right\}\right] e^{-\hat{\jmath} \omega \tau} .
\end{aligned}
$$

Taking the partial derivative of the above equation with respect to $\tau$, we get

$$
\frac{\partial \boldsymbol{t}_{k}\left(e^{\hat{\jmath} \omega}\right)}{\partial \tau}=(-\hat{\jmath} \omega)\left[\left|X_{(1,1)}\right|^{2}+\left|X_{(1,2)}\right|^{2}\right.
$$

$$
\left.+2 \Re\left\{X_{(1,1)} X_{(1,2)}^{*} e^{-\hat{\jmath} \omega \Delta \tau}\right\}\right] e^{-\hat{\jmath} \omega \tau} .
$$

The magnitude squared of the above term is

$$
\begin{aligned}
\left|\frac{\partial \boldsymbol{t}_{k}\left(e^{\hat{\jmath} \omega}\right)}{\partial \tau}\right|^{2}= & {\left[\left|X_{(1,1)}\right|^{4}+\left|X_{(1,2)}\right|^{4}\right.} \\
& \left.+4\left|X_{(1,1)}\right|^{2}\left|X_{(1,2)}\right|^{2}\right] \omega^{2} \\
& +4 \Re\left\{X_{(1,1)} X_{(1,2)}^{*} e^{-\hat{\jmath} \omega \Delta \tau}\right\} \\
& \times\left(\left|X_{(1,1)}\right|^{2}+\left|X_{(1,2)}\right|^{2}\right) \omega^{2} \\
& +2 \Re\left\{\left(X_{(1,1)} X_{(1,2)}^{*}\right)^{2} e^{-\hat{\jmath} \omega \Delta \tau}\right\} \omega^{2} .
\end{aligned}
$$

Substituting (66) in (62) and simplifying in terms of $\beta_{0}^{2}, \beta_{\Delta \tau}^{2}$, and $\beta_{2 \Delta \tau}^{2}$ as given in (40) and (44), proves Corollary 2.1 .

\section{REFERENCES}

[1] P. S. Naidu, Sensor Array Signal Processing. Boca Raton, FL: CRC Press, 2000.

[2] H. L. van Trees, Optimum Array Processing. New York: Wiley-Interscience, 2002, vol. 4, Detection, Estimation and Modulation Theory.

[3] E. Daeipour, W. D. Blair, and Y. Bar-Shalom, "Bias compensation and tracking with monopulse radars in the presence of multipath," IEEE Trans. Aerosp. Electron. Syst., vol. 33, no. 3, pp. 863-882, Jul. 1997.

[4] S. M. Garber, "High resolution sonar signals in a multipath environment," IEEE Trans. Aerosp. Electron. Syst., vol. AES-2, no. 6-Suppl., pp. 431-440, Nov. 1966

[5] J. H. Derryberry and W. D. Gregg, "On optimizing array reception of multipath," IEEE Trans. Aerosp. Electron. Syst., vol. AES-6, no. 2, pp. 188-199, Mar. 1970.

[6] M. J. D. Rendas and J. M. F. Moura, "Cramer-Rao bound for location systems in multipath environments," IEEE Trans. Signal Process., vol. 39, no. 12 , pp. 2593-2610, Dec. 1991.

[7] M. Hamilton and P. M. Schultheiss, "Passive ranging in multipath dominant environments: Part II-Unknown multipath parameters," IEEE Trans. Signal Process., vol. 41, no. 1, Jan. 1993.

[8] Y. Q. Fu and Y. L. Xia, "DOA imaging algorithm based on TR theory," in Proc. IEEE Ind. Electron. Appl. (ICIEA), May 2007, pp. 2109-2112.

[9] Y. Jin and J. M. F. Moura, "Time reversal detection using antenna arrays," IEEE Trans. Signal Process., vol. 57, no. 4, pp. 1396-1414, Apr. 2009.

[10] Y. Jin, J. M. F. Moura, and N. O'Donoughue, "Time reversal in multiple-input multiple-output radar," IEEE J. Sel. Topics Signal Process., vol. 4, no. 1, pp. 210-225, Feb. 2010.

[11] L. Borcea, G. Papanicolaou, C. Tsogka, and J. Berryman, "Imaging and time reversal in random medium," Inv. Problems, vol. 18, pp. 1247-1279, 2002.

[12] F. K. Gruber, E. A. Marengo, and A. J. Devaney, "Time reversal imaging with multiple signal classification considering multiple scattering between the targets," J. Acoust. Soc. Amer., vol. 115, no. 6, pp. 3042-3047, 2004.

[13] F. Foroozan and A. Asif, "Time reversal ground penetrating radar: Range estimation with Cramer Rao lower bound," IEEE Trans. Geosci. Remote Sens., vol. 48, no. 10, pp. 3698-3708, Oct. 2010.

[14] J. Capon, "High resolution frequency wavenumber spectrum analysis," Proc. IEEE, vol. 57, no. 8, pp. 1408-1418, 1969, ug..

[15] N. J. Roseveare and M. R. Azimi-Sadjadi, "Capon beamspace beamforming for distributed acoustic arrays," SPIE, vol. 6562, pp. 19-21, Apr. 2007.

[16] J. Li, P. Stoica, and Z. Wang, "On robust capon beamforming and diagonal loading," in Proc. IEEE Int. Conf. Acoust., Speech, Signal Process. (IEEE ICASSP), Jun. 2003, vol. 5, pp. 337-340.

[17] S. M. Kay, Fundamentals of Statistical Signal Processing: Estimation Theory. Upper Saddle River, NJ: Prentice-Hall, 1993. 
[18] A. Zeira and A. Nehorai, "Frequency domain Cramer-Rao bound for Gaussian processes," IEEE Trans. Acoust., Speech, Signal Process., vol. 38, no. 6, pp. 1063-1066, Jun. 1990.

[19] P. Whittle, "The analysis of multiple stationary time series," J. Roy. Stat. Soc., vol. 15, pp. 125-139, 1953.

[20] F. Foroozan, A. Asif, and M. Sajjadieh, "On time reversal array imaging algorithms," Comput. Sci. Eng. Dept., York Univ., Tech. Rep., 2009, p. 64, submitted to Proc. IEEE, Dec. 2009.

[21] M. Born and E. Wolf, Principles of Optics, 7th ed. Cambridge, U.K.: Cambridge Univ. Press, 1999.

[22] M. Fink, C. Prada, F. Wu, and D. Cassereau, "Self focusing in inhomogeneous media with time reversal acoustic mirrors," in Proc. IEEE Ultrason. Symp., Oct. 1989, vol. 2, pp. 681-686.

[23] M. Fink, "Time reversed acoustics," Scientif. Amer., vol. 281, no. 5, pp. 91-98, Nov. 1999.

[24] D. B. Williams and D. H. Johnson, "Using the sphericity test for source detection with narrow band passive arrays," IEEE Trans. Acoust., Speech, Signal Process., vol. 38, pp. 2008-2014, Nov. 1990.

[25] H. Akaike, "A new look at the statistical model identification," IEEE Trans. Autom. Control, vol. AC-19, pp. 716-723, Dec. 1974.

[26] J. Rissanen, "Modeling by shortest data description," Automatica, vol. 14, pp. 465-471, Sep. 1974.

[27] E. Fishler and H. V. Poor, "Estimation of the number of sources in unbalanced arrays via information theoretic criteria," IEEE Trans. Signal Process., vol. 53, no. 9, pp. 3543-3553, Sep. 2005.

[28] S. Gezici and Z. Sahinoglu, "Ranging in a single-input multiple-output (SIMO) system," IEEE Commun. Lett., vol. 12, no. 3, pp. 197-199, 2008.

[29] Y. Jin, N. O'Donoughue, and J. M. F. Moura, "Position location by time reversal in communication networks," in Proc. IEEE Int. Conf. Acoust., Speech, Signal Process. (ICASSP), Mar. 31-Apr. 4 2008, pp. 3001-3004.

[30] B. R. Mahafza, Radar Signal Analysis and Processing Using Matlab. London, U.K.: Chapman \& Hall-CRC, 2008, ch. 7, pp. 315-352.

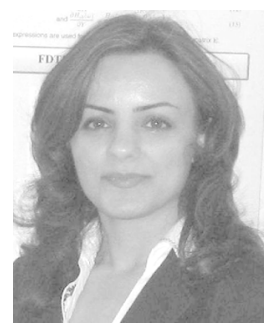

Foroohar Foroozan (S'06) received the B.S. degree from Sharif University of Technology, Tehran, Iran, in 1995, the M.Sc. degree from Tehran Polytechnic, Iran, in 1998, respectively, both in electrical engineering, and the Ph.D. degree in computer science and engineering from York University, Toronto, Canada, in 2011.

From 1998 to 2005, she was working as a Senior Research Engineer in Sharif Advanced Information and Communication Technology Center, Tehran, Iran, and also in semiconductor industry working on data communication chip design. She is currently a Postdoctoral Fellow and instructor at the Faculty of Engineering and Applied Science at the University of Ontario Institute of Technology (UOIT), Oshawa, Canada. Her research interests include statistical signal processing, array processing, time reversal, ultrasonic array imaging, and compressive sensing.

Dr. Foroozan was the recipient of the postgraduate scholarship from the Natural Science and Engineering Research Council (NSERC) of Canada (2007-2009) and the Ontario Graduate Scholarship (OGS) award (2006 and 2009).

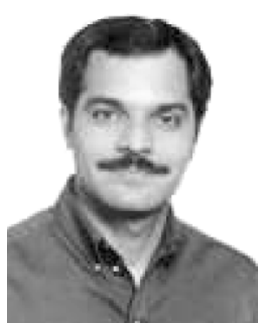

Amir Asif (M'97-SM'02) received the M.Sc. and $\mathrm{Ph} . \mathrm{D}$. degrees in electrical and computer engineering from Carnegie Mellon University (CMU), Pittsburgh, PA, in 1993 and 1996, respectively.

Previously, he was on the faculty of CMU, where he was a Research Engineer from 1997 to 1999 and the Technical University of British Columbia, Vancouver, BC, Canada, where he was an Associate Professor from 1999 to 2002. Since 2002, he has been an Associate Professor of computer science and engineering at York University, Toronto, ON, Canada. He works in the area of statistical signal processing and communications. His current projects include time reversal; distributed signal processing for sensor networks; genomic signal processing; and sparse, block-banded matrix technologies. He has authored over 75 technical contributions, including invited ones, published in international journals and conference proceedings, and the textbook Continuous and Discrete Time Signals and Systems published by the Cambridge University Press.

Dr. Asif has been a Technical Associate Editor for the IEEE SIGNAL PROCESSING LETTERS from 2002 to 2006 and from 2009 to present). He has organized two IEEE conferences on signal processing theory and applications and served on the technical committees of several international conferences. He has received several distinguishing awards, including the York University Faculty of Graduate Studies Teaching Award in 2009; York University Wide Teaching Award (Full-Time Senior Faculty Category) in 2008 from York University; the FSE Teaching Excellence Award (Senior Faculty Category) from York's Faculty of Science and Engineering in 2004 and in 2006; and the CSE Mildred Baptist Teaching Excellence Award from York's Department of Computer Science and Engineering in 2006 and in 2003. He is a member of the Professional Engineering Society of Ontario. 